\title{
The core dominance parameter of extragalactic radio sources ${ }^{\star}$
}

\author{
J. H. Fan and J. S. Zhang \\ Center for Astrophysics, Guangzhou University, Guangzhou 510400, PR China \\ e-mail: fjh@gzhu.edu.cn \\ Received 20 January 2003 / Accepted 12 June 2003 \\ Abstract. In this paper, based on a paper by Liu and Zhang (2002), we have chosen a sample of 542 extragalactic sources \\ (27 BL Lac objects, 300 galaxies (radio galaxies and Seyfert galaxies), and 215 quasars), for which we have calculated the \\ core-dominance parameters and investigated the relation between core-dominance parameter and the core and extended lu- \\ minosities. The core-dominance parameter of galaxies is smaller than that in quasars, which is smaller than that in BL Lac \\ objects. $\log R=-1.40 \pm 0.74$ for galaxies, $\log R=-0.53 \pm 0.92$ for quasars, and $\log R=0.01 \pm 0.65$ for BL Lac objects on \\ average respectively. For quasars, there is clear correlation between the core-luminosity and core-dominance parameter and an \\ anti-correlation between the extended luminosity and the core-dominance parameter, which is explained by the beaming effect.
}

Key words. BL Lacertae objects: general - quasars: general - galaxies: Seyfert - galaxies: jets

\section{Introduction}

The relativistic beaming model has been successfully used to explain observational properties of active galactic nuclei (AGNs). In this model the emissions are composed of two components, namely, the boosted and the isotropic extended ones. The ratio of the two parts is defined as the coredominance parameter (e.g., Orr \& Brown 1982). Some authors used the ratio of flux densities but some others used the ratio of luminosities to indicate the core-dominance parameter (e.g., Punsly et al. 1995, and reference therein), so $R=\frac{S_{\mathrm{C}}}{S_{\mathrm{Ext}}}\left(\right.$ or $\left.R=\frac{L_{\mathrm{C}}}{L_{\mathrm{Ext}}}\right)$. In the unified model, different viewing angles (the angle between the jet and the line of sight) give rise to different subclasses of objects, such as BL Lac objects (BLs), core-dominated quasars (CDQs), lobe-dominated quasars (LDQs), highly polarized quasars (HPQs), and superluminal sources (SLS) etc. BL Lacertae objects are presumed to be FRI type radio galaxies (Fanaroff \& Riley 1974; Urry et al. 1991), but some evidence also suggests that the parent population of BL Lacs should be a mix of FRI and FRII (Kollgaard et al. 1996) and the extended radio morphologies of BLs were found to be of both FRI and II types (Kollgaard et al. 1992); some line emission from FRIIs is weak enough to be BL-like (Laing 1994), and from the Hubble relation point of view, the infrared magnitude of BLs, FRI and FRII(G) fit the same relation (Fan et al. 1997 and reference therein). Thus it may be appropriate to unify BL objects more generally with radio galaxies. Since emissions in jets are strongly beamed, the coredominance parameter, $R$ should indicate the orientation of the jet. Observations also show that optical polarization $\left(P_{\mathrm{opt}}(\%)\right)$

Send offprint requests to: Dr. J. H. Fan,

e-mail: fjh@gzhu.edu.cn

* Table 1 is only available in electronic form at http://www.edpsciences.org is associated with $R$ with high polarization corresponding to high $R$ (Wills et al. 1992; Fan 2002a,b).

Some authors have investigated the relation between $R$ and flux (or luminosities) or the luminosity properties from various samples. For instance, Punsly (1995) investigated the extended luminosity of strong radio cores for a sample of 134 ultraluminous radio core quasars. Those quasars have ultraluminous radio cores. (The core luminosity $P_{\mathrm{c}}>10^{46} \mathrm{erg} \mathrm{s}^{-1}$ in the quasar rest frame from $10 \mathrm{MHz}$ to $250 \mathrm{GHz}$.) Murphy et al. (1993) studied a complete sample of 89 powerful core-dominated radio sources (70 quasars, 19 BL Lacs) with core flux density, $S_{\mathrm{c}}^{5 \mathrm{GHz}}>1 \mathrm{Jy}$ at $5 \mathrm{GHz}$. They visited the difference of coredominance parameter, redshift, extend radio luminosity. Hough et al. (1989) defined a complete sample of 28 double-lobed radio quasars to make a statistical analysis of the large-scale structure to check for consistency with the beaming hypothesis in the central components. Hutchings et al. (1988), Neff et al. (1989), Neff \& Hutchings (1990) studied a large sample of 250 core-dominated quasars. It was shown that the core dominance parameter increases with redshift. Very recently, a larger sample of 661 extragalatic radio sources were compiled and a relation between the $5 \mathrm{GHz}$ core luminosity and $1.4 \mathrm{GHz}$ total luminosity was discussed (Liu \& Zhang 2002). From their paper we chose a sample of 542 sources with both core and total luminosities, and calculated their core-dominance parameters. The $\log R$ distribution and the correlation of luminosity and $R$ are investigated. In Sect. 2, we give the sample and some results. In Sect. 3, we present some discussions and a brief summary.

\section{Sample and results}

\subsection{Sample}

From the paper by Liu \& Zhang (2002), a sample of $542 \mathrm{ob-}$ jects with total luminosity (in $\mathrm{W} \mathrm{Hz}^{-1}$ ) at $1.4 \mathrm{GHz}$ and core 
luminosity at $5 \mathrm{GHz}$ were chosen. Out of them, 27 are BL Lac objects, 300 are galaxies, and 215 are quasars. They are listed in Table 1, in which Col. 1 gives the source name, Col. 2 identification (BL stands for BL Lacertae objects, Q for quasars, $\mathrm{G}$ for radio galaxy, S0.5, S1, S2 and S3 for Seyfert galaxies), Col. 3 redshift, Col. 4 total luminosity at $1.4 \mathrm{GHz}$ in units of W Hz${ }^{-1}$, Col. 5 core luminosity at $5 \mathrm{GHZ}$ in units of $\mathrm{W} \mathrm{Hz}{ }^{-1}$, Col. 6 for core dominance parameter corresponding to $\alpha_{\mathrm{E}}=0.5$, and Col. 7 for core dominance parameter corresponding to $\alpha_{\mathrm{E}}=1.0$.

\subsection{Result}

To calculate the core-dominance parameter at $5 \mathrm{GHz}$, we assume that the core spectral index is $\alpha_{\mathrm{C}}=0.0\left(S_{v} \propto v^{-\alpha}\right)$, and the extended spectral index is $\alpha_{\mathrm{E}}=0.5$ and 1.0 respectively. Therefore, we have that the $1.4 \mathrm{GHz}$ core luminosity is equal to the $5 \mathrm{GHz}$ core luminosity, $P_{1.4 \mathrm{GHz}}^{\mathrm{C}}=P_{5 \mathrm{GHz}}^{\mathrm{C}}$, and the $1.4 \mathrm{GHz}$ extended luminosity is the difference of the $1.4 \mathrm{GHz}$ total and core luminosities, $P_{1.4 \mathrm{GHz}}^{\mathrm{E}}=P_{1.4 \mathrm{GHz}}^{\mathrm{T}}-P_{5 \mathrm{GHz}}^{\mathrm{C}}$, considering the cosmic expansion, we have $5 \mathrm{GHz}$ extended luminosity $P_{\mathrm{E}}=P_{5 \mathrm{GHz}}^{\mathrm{E}}(1+z)^{\left(\alpha_{\mathrm{E}}-1\right)}=P_{1.4 \mathrm{GHz}}^{\mathrm{E}} \times(1.4 / 5)^{\alpha_{\mathrm{E}}}(1+z)^{\left(\alpha_{\mathrm{E}}-1\right)}$. In this sense, the core-dominance parameter at $5 \mathrm{GHz}$ is

$R=\frac{P_{\mathrm{C}}}{P_{\mathrm{E}}}=\frac{P_{5 \mathrm{GHz}}^{\mathrm{C}}}{P_{1.4 \mathrm{GHz}}^{\mathrm{T}}-P_{5 \mathrm{GHz}}^{\mathrm{C}}}(1.4 / 5)^{-\alpha_{\mathrm{E}}}(1+z)^{-\alpha_{\mathrm{E}}}$.

The obtained results are listed in Cols. 6 and 7 for $\alpha_{\mathrm{E}}=$ 0.5 and 1.0 respectively. The correspoding distribution is also shown in Fig. 1.

\subsection{1. $R$ distribution}

$\log R$ histogram of the whole sample are shown in Fig. 1 for the differently extended spectral index, $\alpha_{\mathrm{E}}$. The upper panel of Fig. 1 corresponds to $\alpha_{\mathrm{E}}=0.5$ while the lower panel to $\alpha_{\mathrm{E}}=1.0$. We found that the distribution of $\log R$ obtained from different extended spectral indices $\left(\alpha_{\mathrm{E}}\right)$ is different. The steeper the spectrum the larger the $R$ as shown in Fig. 1. If we consider different type of sources separately, we find that, on average, the core-dominance parameter of galaxies is smaller than that in quasars, which is smaller than that in BL Lac objects with the average $\log R$ for $\alpha_{\mathrm{E}}=0.5$ case being as follows: $\log R=$ $-1.40 \pm 0.74$ for galaxies, $\log R=-0.53 \pm 0.92$ for quasars, and $\log R=0.01 \pm 0.65$ for BL Lac objects respectively.

\subsubsection{Relation between $R$ and core and extended luminosities}

From a statistical analysis, we consider the correlation between $\log R$ and $\log P_{\mathrm{C}}$ and $\log P_{\mathrm{E}}$ for the whole sample and individual sub-samples respectively. (Here we only take into account the $\alpha_{\mathrm{E}}=0.5$ case.) The corresponding diagrams are shown in Figs. 2 to 5. Relations between $R$ and $P_{\mathrm{E}}$ and $P_{\mathrm{C}}$ for the whole sample are shown in Fig. 2. When a linear regression is applied to the data, we find a clear correlation between $\log R$ and $\log P_{\mathrm{C}}$ for the whole sample, $\log P_{\mathrm{C}}=(0.96 \pm 0.07) \log R+$ $25.34 \pm 0.10$ with a correlation coefficient $r=0.50$ and a chance

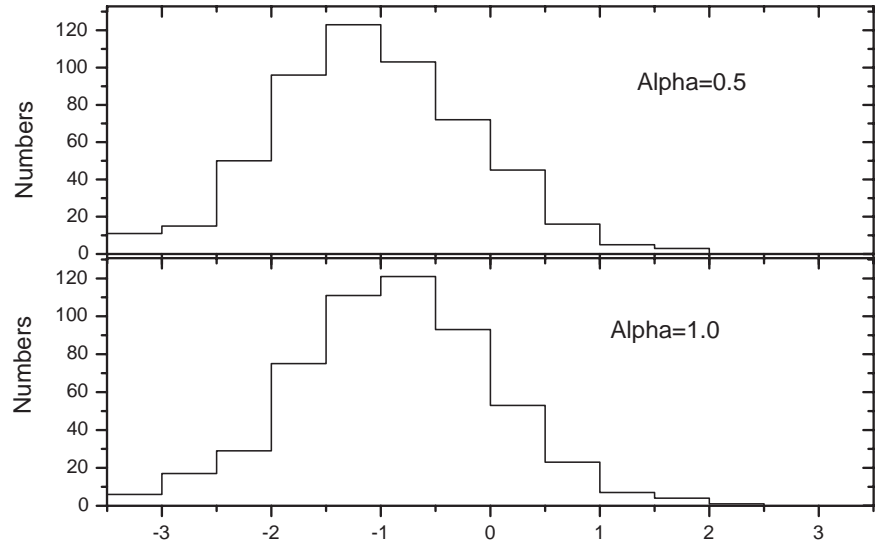

Fig. 1. Histogram of $\log R$ for the whole sample with differently extended spectral index. The upper panel corresponds to $\alpha_{\mathrm{E}}=0.5$, the lower panel to $\alpha_{\mathrm{E}}=1.0$.
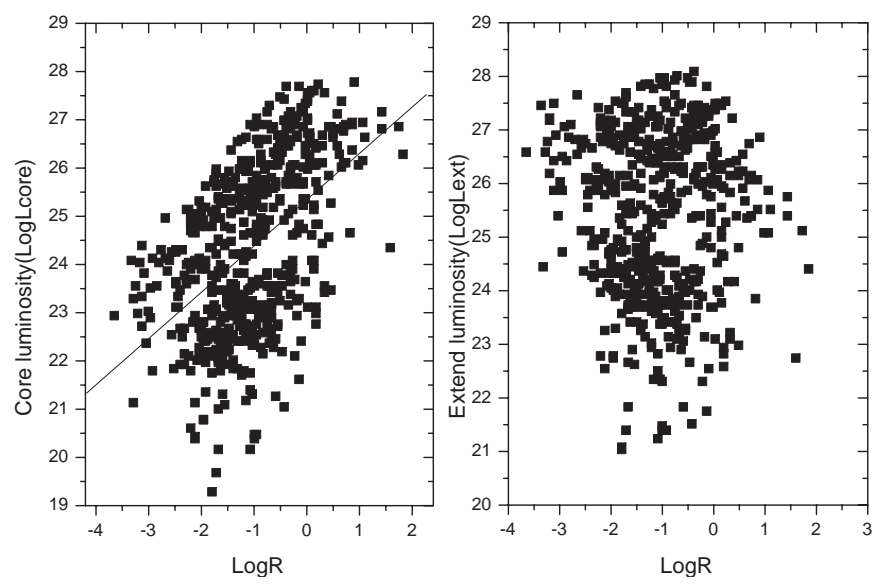

Fig. 2. Relation of luminosity and the core-dominance parameter (left panel is for core-luminosity and the straight line represents the best fit result and the right panel is for extended luminosity) for the whole sample.

probability $p \sim 0$. However, there is no clear relation for $\log R$ and $\log P_{\mathrm{E}}$. We also consider the relation for different subclasses. The relation between $R$ and $P_{\mathrm{E}}$ and $P_{\mathrm{C}}$ for quasars is shown in Fig. 3. There is a clear correlation between $\log R$ and $\log P_{\mathrm{C}}$ for quasars, $\log P_{\mathrm{C}}=(0.64 \pm 0.06) \log R+26.4 \pm 0.06$ with a correlation coefficient $r=0.59$ and a chance probability $p \sim 0$, and an anti-correlation for $\log R$ and $\log P_{\mathrm{E}}$, $\log P_{\mathrm{E}}=-(0.34 \pm 0.05) \log R+26.43 \pm 0.06$ with a correlation coefficient $r=-0.39$ and a chance probability $p=10^{-7}$. The relation between $R$ and $P_{\mathrm{E}}$ and $P_{\mathrm{C}}$ for BL Lacs is shown in Fig. 4. There is no clear correlation between $\log R$ and $\log P_{\mathrm{C}}$ or for $\log R$ and $\log P_{\mathrm{E}}$ because of the small sample. The relation between $R$ and $P_{\mathrm{E}}$ and $P_{\mathrm{C}}$ for galaxies is shown in Fig. 5. There is an anti-correlation between $\log R$ and $\log P_{\mathrm{E}}$, $\log P_{\mathrm{E}}=-(0.87 \pm 0.08) \log R+(23.26 \pm 0.14)$ with a correlation coefficient $r=-0.50$ and a chance probability $p \sim 0$, but there is no clear relation for $\log R$ and $\log P_{\mathrm{C}}$. 

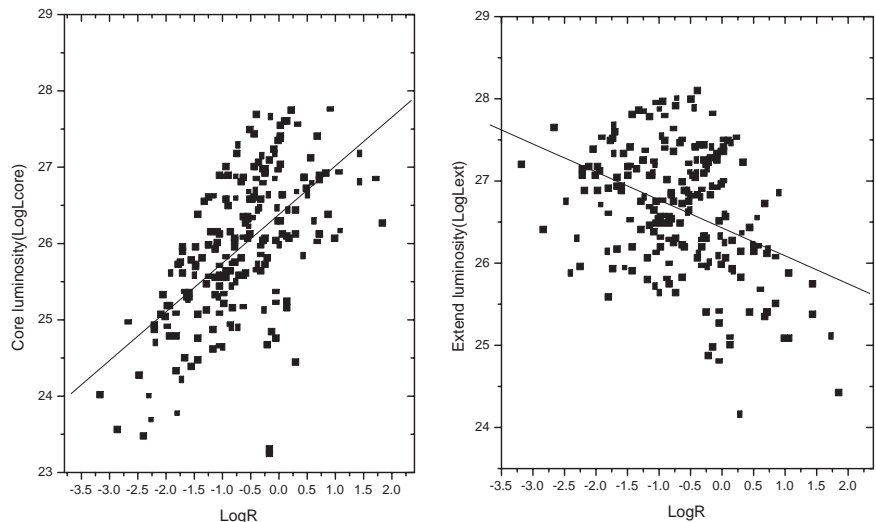

Fig. 3. Relation between luminosity and core-dominance parameter (left panel is for core-luminosity and the right panel is for extended luminosity) for quasars. The straight lines represent the best fit results.
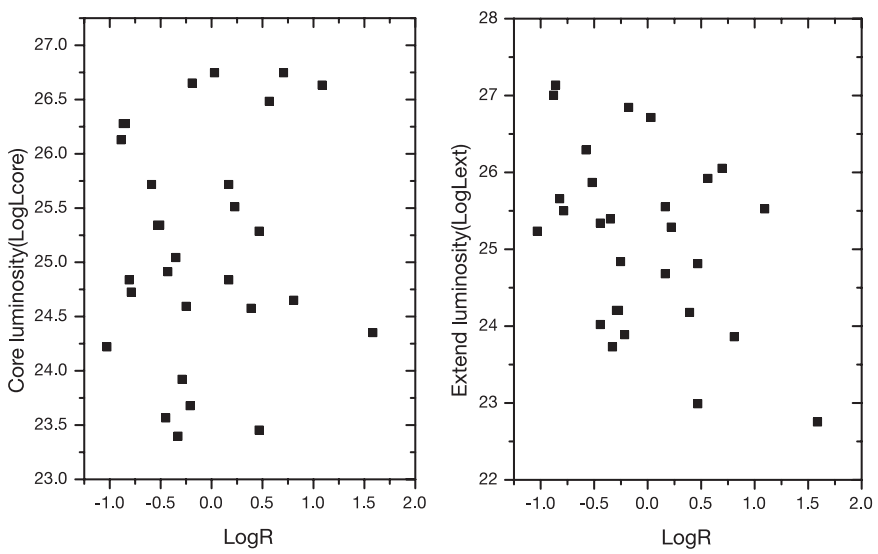

Fig. 4. Relation between luminosity and core-dominance parameter (left panel is for core-luminosity and the right panel is for extended luminosity) for BL Lacertae objects.

\subsubsection{Relation between $R$ and redshift}

From relation (1), it is expected that there is a relation between redshift and core-dominance parameter, $R$, since $R=\frac{P_{\mathrm{C}}}{P_{\mathrm{E}}}=$ $\frac{P_{5 \mathrm{GHz}}^{\mathrm{C}}}{P_{1.4 \mathrm{GHz}}^{\mathrm{T}}-P_{5 \mathrm{GHz}}^{\mathrm{C}}}(1.4 / 5)^{-\alpha_{\mathrm{E}}}(1+z)^{-\alpha_{\mathrm{E}}}$. From our calculation and the known redshift, we find that there is no clear correlation between $R$ and redshift (see Fig. 6).

\section{Discussion}

In extragalactic radio sources, the core-dominance parameter is taken as an orientation parameter. Wills et al. (1992) compiled a quasar sample. Ghisellini et al. (1993) also compiled a sample of extragalactic sources and found that the average $\log R$ in BL Lacs is larger than that in flat spectrum radio quasars (FSRQs). However, Murphy et al. (1993) obtained a distribution of $\log R$ for a core-dominated radio source sample (56 quasars, 18 BL Lacs) with the $5 \mathrm{GHz}$ core flux densities being greater than $1 \mathrm{Jy}$. They found that there is a tendency for BL Lacs to have lower $R$ than quasars in their sample. Although the difference is not significant, the results do not support the argument that BL Lacs are quasars with more highly beamed cores. Our present results show a tendency that the BL Lacs
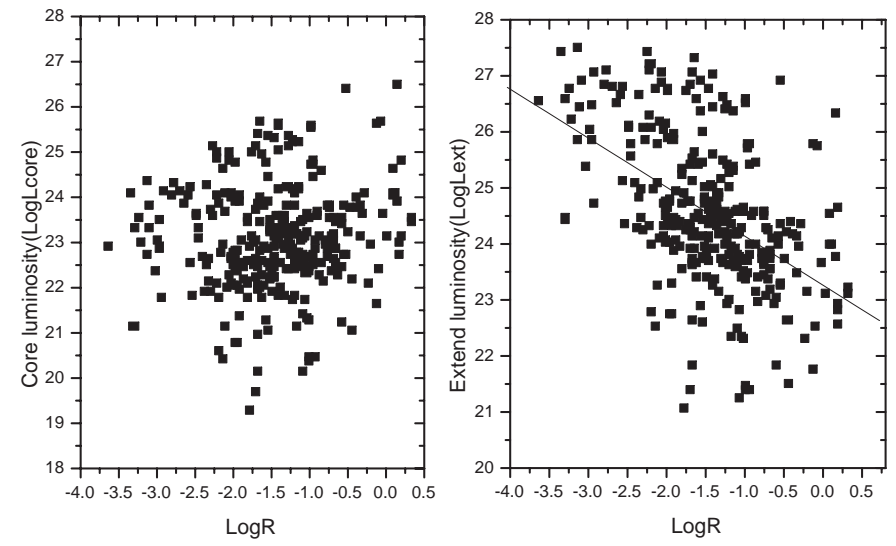

Fig. 5. Relation between luminosity and core-dominance parameter (left panel is for core-luminosity and the right panel is for extended luminosity, the straight line represents the best fit result) for galaxies.
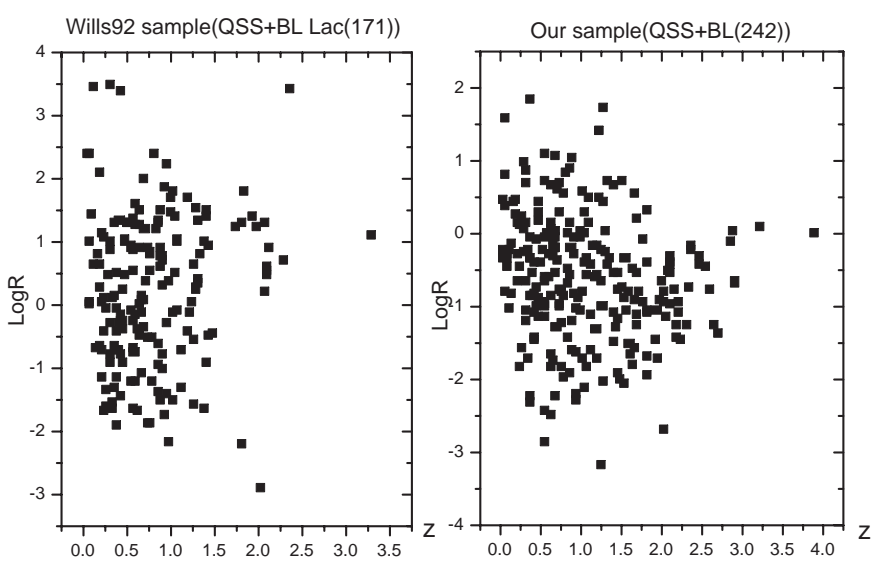

Fig. 6. Relation between the core-dominance parameter and redshift (left panel is for the data from Wills et al. (1992), and the right panel is from our results for BLs and quasars.

have higher $R$ than quasars, and that quasars have higher $R$ than galaxies. This difference is illustrated by comparing their distribution of $\log R$ (Figs. 7, 9, 11) and cumulative probability (K-S test in Figs. 8, 10 and 12) for BL Lacs and galaxies, quasars and galaxies, BL Lacs and quasars respectively. The K-S test results indicate that the null hypothesis (they both are from the same parent population) cannot be rejected at the following confidence level for the different samples: $p=0.003$ for BL Lacs and quasars, $p<10^{-4}$ for BL Lacs and galaxies, and $p<10^{-4}$ for quasars and galaxies. So, both BL Lacs and quasars are different from galaxies in this respect.

The $R$ difference between different subclasses found here is consistent with that found by Ghisellini et al. (1993) but is in conflict with that found in Murphy et al. (1993). This difference is from the fact that Murphy et al. only considered bright sources $\left(S_{5 \mathrm{GHz}}>1 \mathrm{Jy}\right)$ while the sources considered in the paper by Ghisellini et al. (1993) and those in the present paper cover a large range of flux densities. Very recently, we found that the apparently higher $R$ in BL Lacs than in FSRQs does not imply that the boosting factor (Doppler factor) in BL Lacs is greater than that in FSRQs. It is caused by the ratio of the intrinsic flux density in the jet to the extend flux density in the 


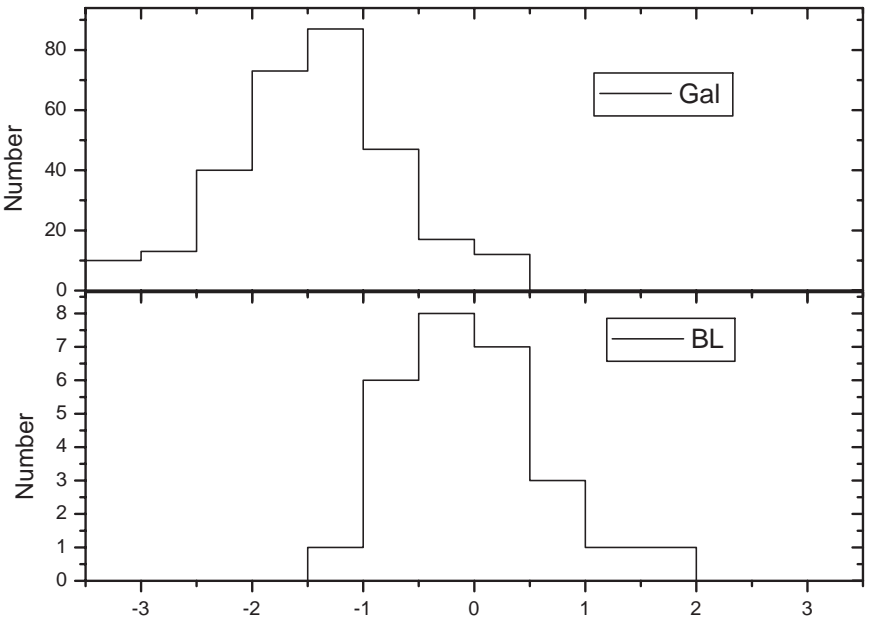

Fig. 7. Histogram comparison of core-dominance parameter for galaxies (upper panel) and BL Lacs (lower panel).

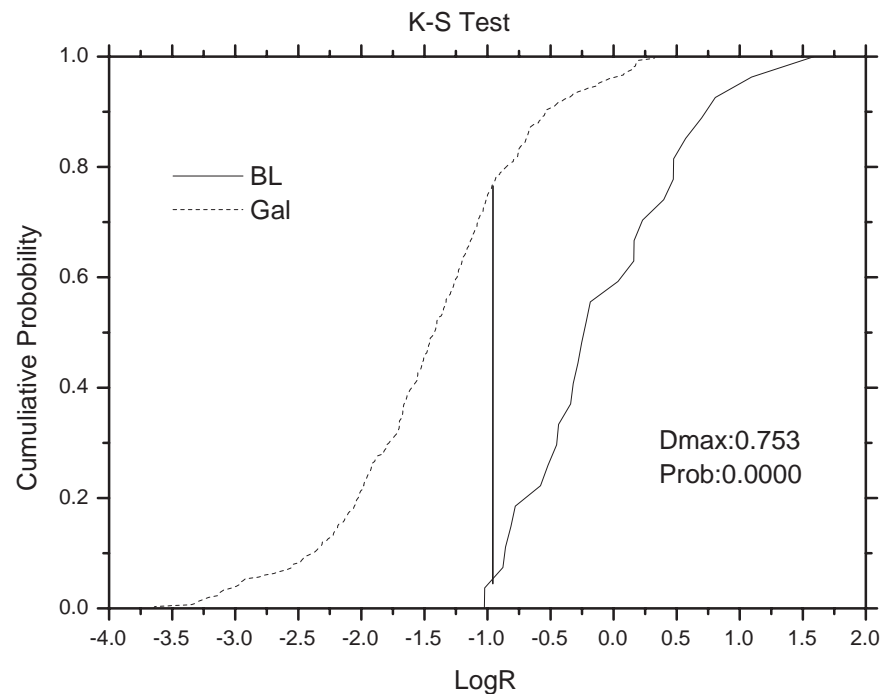

Fig. 8. K-S test for BL Lacs and galaxies.

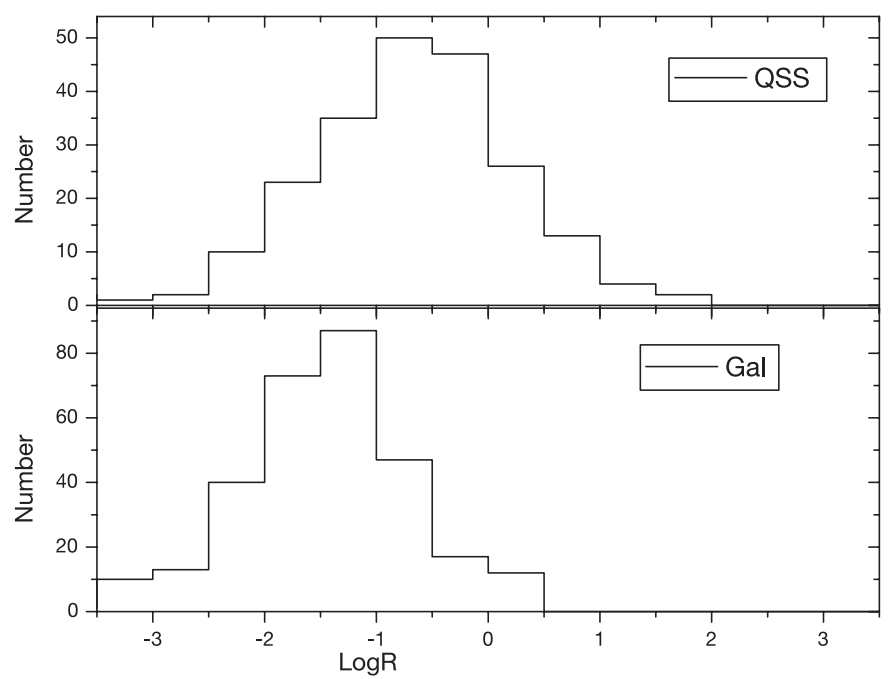

Fig. 9. Histogram comparison of core-dominance parameter for quasars (upper panel) and galaxies (lower panel).

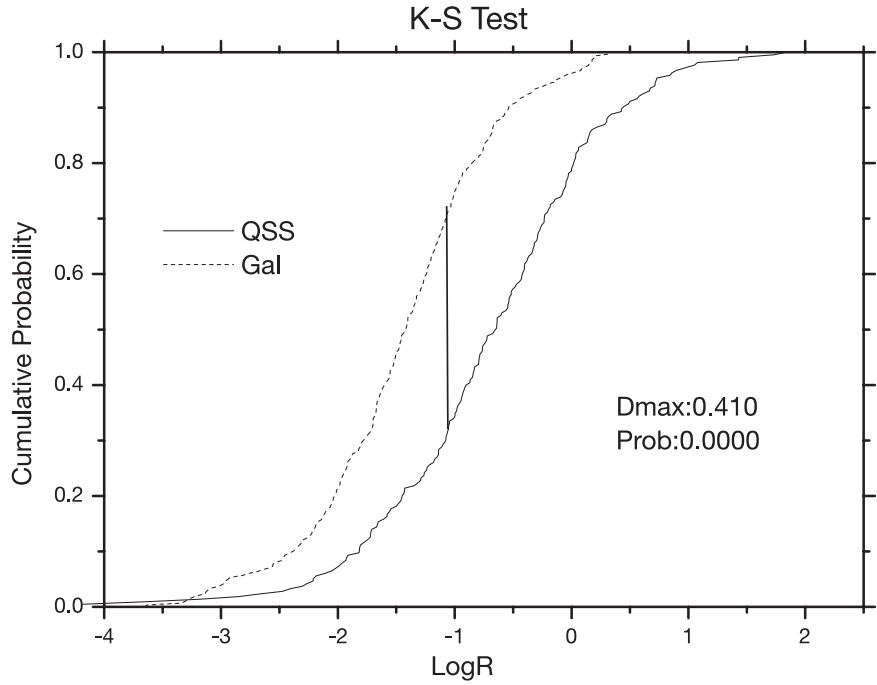

Fig. 10. K-S test for quasars and galaxies.

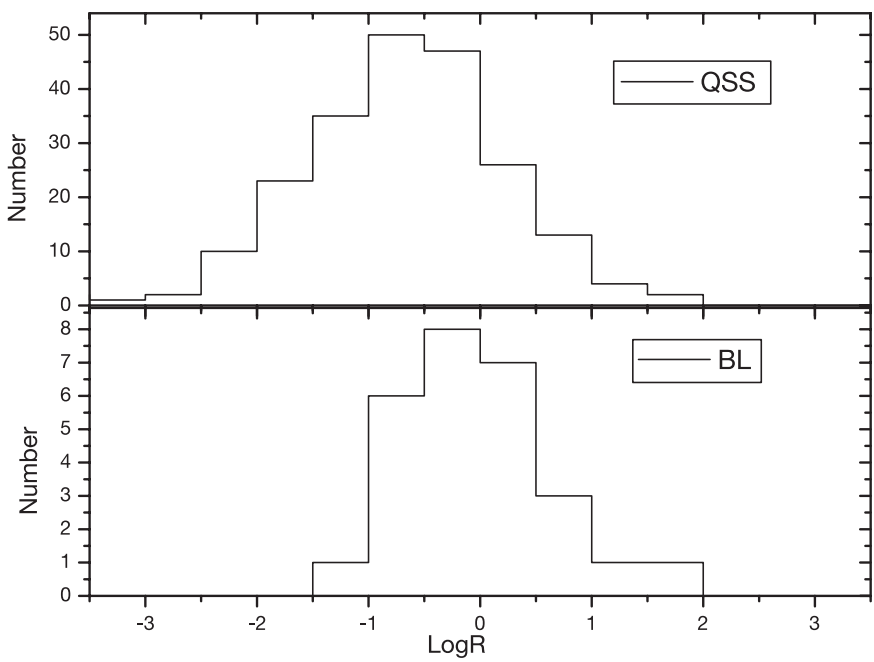

Fig. 11. Histogram comparison of core-dominance parameter for quasars (upper panel) and BL Lacs (lower panel).

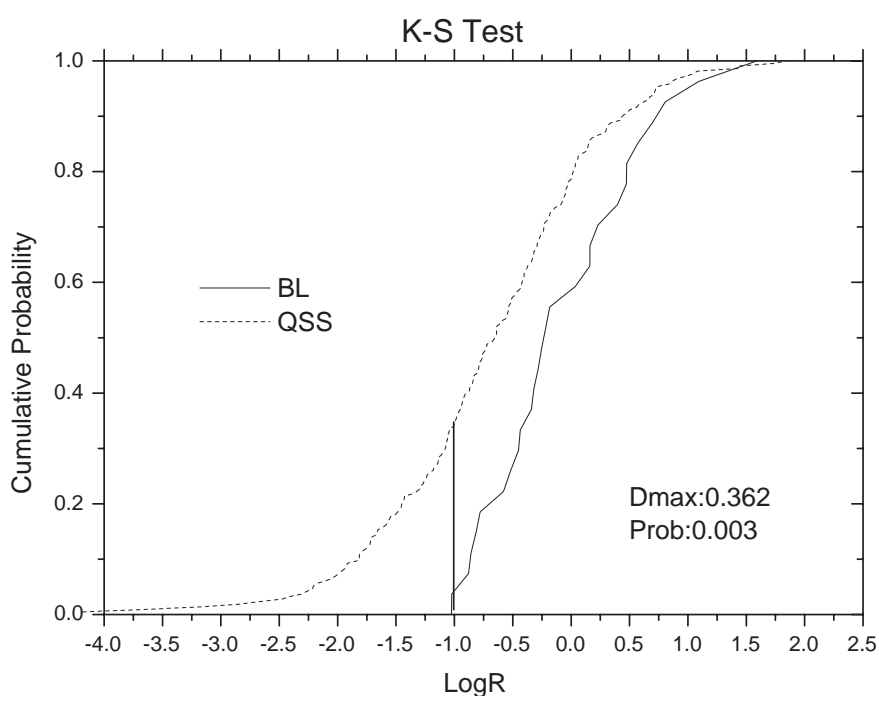

Fig. 12. K-S test for BL Lacs and quasars. 
co-moving frame, $f=\frac{S_{\text {core }}^{\text {in }}}{S_{\text {ext }}^{\text {in }}}$, which is greater in BL Lacs than that in FSRQs, namely $f_{\mathrm{BLs}}>f_{\mathrm{FSRQs}}$. This difference in $f$ between BLs and FSRQs can explain the emission line difference in BL Lacs and FSRQs (Fan 2002a,b, 2003).

\subsection{Beaming model and R-luminosity relation for quasars}

From Fig. 3, we have relations between $\log R$ and $\log P_{\mathrm{C}}$ and $\log P_{\mathrm{E}}$ for quasars. Similar correlations were also discussed in previous papers using various samples (Hough et al. 1989; Murphy et al. 1993; Punsly 1995). From their work, we found that $\log P_{\mathrm{E}}$ is anti-correlated with $\log R$ for core-dominated radio quasars $(\log R>0)$, but there is no obvious correlation between $\log P_{\mathrm{E}}$ and $\log R$ for lobe-dominated radio quasars $(\log R<0)$. No clear correlation can be found between $\log S_{\mathrm{C}}$ (or $\log P_{\mathrm{C}}$ ) and $\log R$ if we consider the data given in the papers by Murphy et al. (1993) and Punsly (1995). However, a clear correlation can be found between $\log S_{\mathrm{C}}$ (or $\log P_{\mathrm{C}}$ ) and $\log R$ if we consider the data given in the papers by Hough \& Readhead (1989). Why is there such a contradiction? In fact, this different result can be explained using a beaming model as shown below. From a two-component beaming model, we have $R=\frac{P_{\mathrm{C}}}{P_{\mathrm{E}}}$, which is used in the following luminosity- $R$ relation consideration.

Firstly, we consider the $\log R-\log P_{\mathrm{C}}$ relation. $R$ can be expressed in a form

$\frac{R}{1+R}=\frac{P_{\mathrm{C}}}{P_{\mathrm{E}}+P_{\mathrm{C}}}=\frac{P_{\mathrm{C}}}{P_{\mathrm{T}}}$

If $P_{\mathrm{T}}$ is a constant, then $R$ is proportional to $P_{\mathrm{C}}$ when $R$ is much smaller than unity, and there is no correlation between $R$ and $P_{\mathrm{C}}$ when $R$ is much greater than unity. Therefore, there is no $\log R-$ $\log P_{\mathrm{C}}$ relation when $R$ is large and there is $\log R-\log P_{\mathrm{C}}$ relation when $R$ is small. When the data $(\log R<0$ for all but two objects) by Hough \& Readhead (1989) are considered, there is a clear correlation between $\log P_{\mathrm{C}}$ and $\log R$. From the data in the present paper we have $\log P_{\mathrm{T}}=26.86 \pm 0.67$ for quasars. When we use $\log P_{\mathrm{T}}=26.86+0.67=27.53$ and $\log P_{\mathrm{T}}=26.86-0.67=26.19$, we can get two curves that show the relation between $\log P_{\mathrm{C}}$ and $\log R$ as shown in Fig. 13. It is clear that most of the observation data are in between the area bounded by the two curves.

Secondly, we consider the $\log R-\log P_{\mathrm{E}}$ relation. $R$ can be expressed in a form

$1+R=\frac{P_{\mathrm{C}}+P_{\mathrm{E}}}{P_{\mathrm{E}}}=\frac{P_{\mathrm{T}}}{P_{\mathrm{E}}}$.

If $P_{\mathrm{T}}$ is a constant, then $R+1$ is anti-proportional to $P_{\mathrm{E}}$ when $R$ is much larger than unity, and there is no correlation between $R$ and $P_{\mathrm{E}}$ when $R$ is much smaller than unity. Therefore, there is no $\log R-\log P_{\mathrm{E}}$ relation when $R$ is small and there is an anti-correlation of $\log R-\log P_{\mathrm{E}}$ when $R$ is large. When the data ( $\log R>0.5$ for all sources) by Punsly (1995) are considered, a clear anti-correlation is seen between $\log P_{\mathrm{E}}$ and $\log R$. When we use $\log P_{\mathrm{T}}=27.53$ and $\log P_{\mathrm{T}}=26.19$, we can get two curves to show the relation between $\log P_{\mathrm{E}}$ and $\log R$ as shown
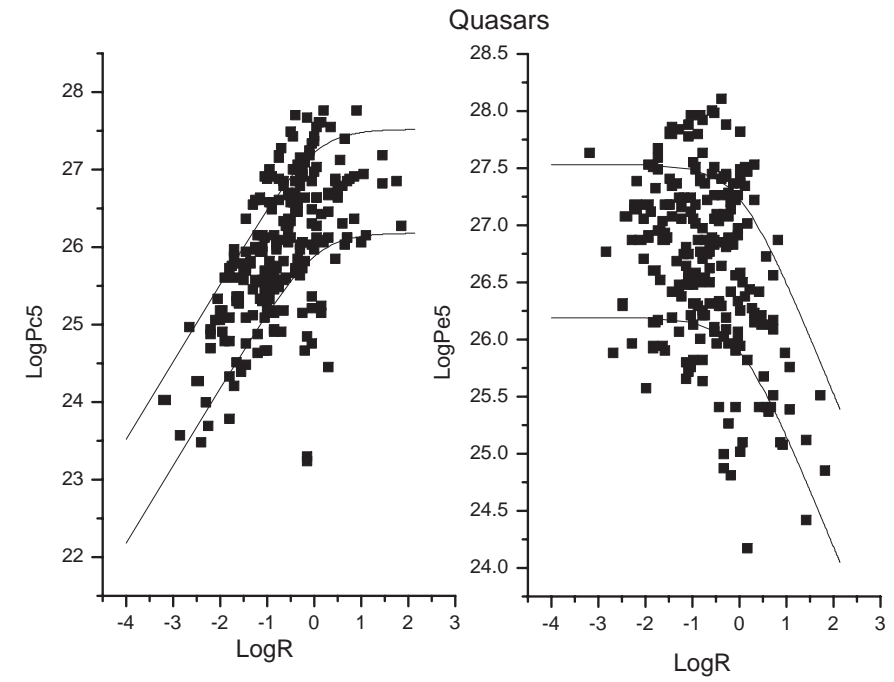

Fig. 13. Plot of luminosity against core-dominance parameter (left panel is for core-luminosity, the right panel is for extended luminosity) for quasars. The curves represent $\log P_{\mathrm{T}}=27.53 \mathrm{~W} \mathrm{~Hz}^{-1}$ (upper curve) and $\log P_{\mathrm{T}}=26.19 \mathrm{~W} \mathrm{~Hz}^{-1}$ (lower curve).

in Fig. 10, in which most of the observational data are in between the area bounded by the two curves.

\subsection{Comparison with Wills et al. (1992)}

From relation (1), there should be a correlation between $R$ and redshift, but our calculation shown in Fig. 6 (right panel) does not show any correlation. This is probably from the fact that $R$ in relation (1) does not only depends on redshift but also

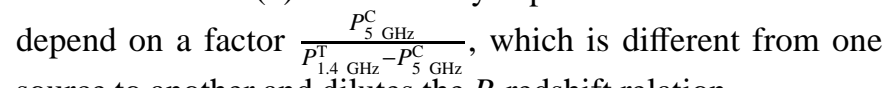
source to another and dilutes the $R$-redshift relation.

In 1992, Wills et al. compiled core-dominance parameters for a large sample of extragalactic sources including BL Lacs and quasars, and exammed the $R$-redshift relation, but they found no clear $R$-redshift relation. For comparison, we consider their data in Fig. 6 (left panel). The two panels show similar results. Our result is consistent with that by Wills et al. (1992). Therefore, double jet radio sources (our results) and single jet radio sources show no difference in the $R$-redshift relation.

\subsection{Summary}

In the present paper, we calculated the core-dominance parameters $(R)$ for a sample of 542 sources and compared the $R$ values for different sub-samples (BL Lacs, Quasars, and galaxies). The average $R$ in BL Lacs is larger than that in quasars, whose average $R$ is greater than that in galaxies. We also investigated the correlation between $R$ and luminosity. We found that there is a correlation between core-luminosity and $R$ and an anticorrelation between extended luminosity and $R$ for quasars. This relationship has been explained using a beaming model. Furthermore, there is no clear correlation between $R$ and redshift for the whole sample or for individual sub-samples.

Acknowledgements. This work is partially supported by the National Natural Scientific Foundation of China(19973001), National 973 
project (NKBRSF G19990754), the National Science Fund for Distinguished Young Scholars (10125313), and the Fund for Top Scholars of Guangdong Province (Q02114). The authors thank the referee, Dr. Rick Perley for the valuable comments and suggestions on this work.

\section{References}

Browne, I. W. A., \& Murphy, D. W. 1987, MNRAS, 226, 601 Fan, J. H. 2003, ApJ, 585, L23

Fan, J. H. 2002a, PASJ, 54, L55

Fan, J. H. 2002b, IAU Symp., 214, August 5-10, Suzhou, China

Fan, J. H., Okudaira, A., Lin, R. G., \& Xie, G. Z. 1997, Ap\&SS, 253, 275

Fanaroff, B. L., \& Riley, J. M. 1974, MNRAS, 167, 31

Ghisellini, G., Padovani, P., Celotti, A., \& Maraschi, L. 1993, ApJ, 407, 65

Hough, D. H., \& Readhead, A. C. S. 1989, AJ, 1208, 1225
Hutchings, J. B., Price, R., \& Gower, A. 1988, ApJ, 329, 122

Kollgaard, R. I., Palma, C., Laurent-Muehleisen, S. A., \& Feigelson, E. D. 1996, ApJ, 465, 115

Kollgaard, R. I., Wardle, J. F. C., Roberts, D. H., \& Gabuzda, D. C. 1992, AJ, 104, 1687

Laing, R. A., Jenkins, C. R., Wall, J. V., \& Unger, S. W. 1994, ASP Conf. Ser., 54, 201

Liu, F. K., \& Zhang, Y. H. 2002, A\&A, 381, 757

Murphy, D. W., Browne, I. W. A., \& Perley, R. A. 1993, MNRAS, 264, 298

Neff, S. G., Hutchings, J. B., \& Gower, A. C. 1989, AJ, 97, 1291

Neff, S. G., \& Hutchings, J. B. 1990, AJ, 100, 1441

Orr, M. J. L., \& Brown, I. W. A. 1982, MNRAS, 200, 1067

Punsly, B. 1995, AJ, 109, 1555

Urry, C. M., Padovani, P., \& Stickel, M. 1991, ApJ, 382, 501

Wills, B. J., Wills, D., Breger, M., Antonucci, R. R. J., \& Barvainis, R. 1992, ApJ, 398, 454 
J. H. Fan and J. S. Zhang: The core dominance parameter of extragalactic radio sources, Online Material p. 1

\section{Online Material}


J. H. Fan and J. S. Zhang: The core dominance parameter of extragalactic radio sources, Online Material p. 2

Table 1. Core-dominance parameters.

\begin{tabular}{|c|c|c|c|c|c|c|c|c|c|c|c|c|c|}
\hline $\begin{array}{l}\text { Name } \\
\text { (1) }\end{array}$ & $\begin{array}{l}\text { ID } \\
\text { (2) }\end{array}$ & $\begin{array}{c}z \\
(3)\end{array}$ & $\begin{array}{c}\log P^{T} \\
\text { (4) }\end{array}$ & $\begin{array}{c}\log P^{\mathrm{C}} \\
\text { (5) }\end{array}$ & $\begin{array}{c}\log R \\
\text { (6) }\end{array}$ & $\begin{array}{c}\log R \\
\text { (7) }\end{array}$ & $\begin{array}{l}\text { Name } \\
\text { (1) }\end{array}$ & $\begin{array}{l}\text { ID } \\
\text { (2) }\end{array}$ & $\begin{array}{c}z \\
\text { (3) }\end{array}$ & $\begin{array}{c}\log P^{\mathrm{T}} \\
(4)\end{array}$ & $\begin{array}{c}\log P^{\mathrm{C}} \\
\text { (5) }\end{array}$ & $\begin{array}{c}\log R \\
\text { (6) }\end{array}$ & $\begin{array}{c}\log R \\
\text { (7) }\end{array}$ \\
\hline $0414+009$ & $\mathrm{BL}$ & 0.287 & 25.30 & 24.70 & -0.20 & 0.08 & $0156-252$ & $\mathrm{G}$ & 2.090 & 27.45 & 25.85 & -1.31 & -1.04 \\
\hline $0521-365$ & BL & 0.061 & 25.83 & 24.75 & -0.77 & -0.49 & $0157+393$ & G & 0.072 & 24.34 & 23.66 & -0.30 & -0.03 \\
\hline $0548-322$ & BL & 0.069 & 24.39 & 23.60 & -0.44 & -0.16 & $0206+35$ & G & 0.038 & 24.52 & 23.15 & -1.07 & -0.80 \\
\hline 0723-008 & $\mathrm{BL}$ & 0.130 & 25.99 & 24.89 & -0.79 & -0.51 & 0211-122 & G & 2.336 & 27.30 & 25.30 & -1.72 & -1.44 \\
\hline $0820+225$ & BL & 0.951 & 27.47 & 26.42 & -0.73 & -0.46 & $0219+421$ & G & 0.002 & 21.68 & 20.38 & -1.00 & -0.72 \\
\hline $0826+180$ & BL & 0.089 & 24.61 & 23.96 & -0.26 & 0.01 & $0220+427$ & G & 0.022 & 24.69 & 22.59 & -1.82 & -1.54 \\
\hline $0828+493$ & $\mathrm{BL}$ & 0.548 & 26.73 & 25.90 & -0.48 & -0.21 & $0234+315$ & G & 1.575 & 27.08 & 23.72 & -3.08 & -2.81 \\
\hline $0829+046$ & BL & 0.180 & 25.55 & 25.35 & 0.51 & 0.79 & $0238+085$ & G & 0.021 & 23.80 & 22.54 & -0.96 & -0.68 \\
\hline $0954+658$ & BL & 0.386 & 26.28 & 25.48 & -0.45 & -0.17 & $0247+467$ & G & 0.029 & 23.97 & 22.56 & -1.12 & -0.84 \\
\hline $1011+496$ & BL & 0.200 & 25.25 & 24.91 & 0.20 & 0.48 & $0255+05$ & G & 0.024 & 24.61 & 22.40 & -1.93 & -1.65 \\
\hline $1101+384$ & BL & 0.031 & 23.68 & 23.47 & 0.48 & 0.76 & $0256+132$ & G & 0.075 & 24.07 & 22.30 & -1.49 & -1.21 \\
\hline $1144+352$ & BL & 0.063 & 24.39 & 24.37 & 1.60 & 1.88 & $0258+350$ & G & 0.020 & 23.88 & 22.48 & -1.11 & -0.83 \\
\hline $1156+295$ & BL & 0.729 & 27.10 & 26.99 & 0.82 & 1.09 & $0300+162$ & G & 0.032 & 24.52 & 21.94 & -2.30 & -2.03 \\
\hline $1219+285$ & BL & 0.100 & 25.56 & 24.26 & -1.00 & -0.72 & $0305+039$ & G & 0.029 & 24.83 & 23.77 & -0.74 & -0.47 \\
\hline $1400+162$ & BL & 0.244 & 25.76 & 25.01 & -0.39 & -0.11 & $0313+683$ & G & 0.090 & 24.92 & 23.56 & -1.06 & -0.79 \\
\hline $1413+135$ & $\mathrm{BL}$ & 0.249 & 25.91 & 25.61 & 0.28 & 0.55 & $0314+416$ & G & 0.026 & 24.76 & 22.15 & -2.33 & -2.06 \\
\hline $1511+103$ & $\mathrm{BL}$ & 0.049 & 24.79 & 24.68 & 0.82 & 1.09 & $0319-454$ & G & 0.063 & 25.26 & 22.82 & -2.16 & -1.89 \\
\hline $1652+398$ & $\mathrm{BL}$ & 0.034 & 24.30 & 23.69 & -0.21 & 0.07 & 0320-37 & G & 0.006 & 24.73 & 21.15 & -3.30 & -3.03 \\
\hline $1749+096$ & BL & 0.322 & 26.16 & 25.83 & 0.22 & 0.50 & $0326+396$ & G & 0.024 & 24.06 & 22.70 & -1.06 & -0.79 \\
\hline $1749+701$ & BL & 0.770 & 27.57 & 26.52 & -0.73 & -0.46 & $0327+246$ & G & 0.106 & 23.71 & 22.71 & -0.68 & -0.40 \\
\hline $1803+784$ & BL & 0.680 & 27.34 & 26.97 & 0.15 & 0.42 & 0331-013 & G & 0.139 & 25.77 & 23.96 & -1.53 & -1.25 \\
\hline $1807+698$ & BL & 0.050 & 24.84 & 24.60 & 0.41 & 0.68 & $0335+096$ & G & 0.035 & 23.70 & 22.36 & -1.04 & -0.77 \\
\hline $1826+796$ & BL & 0.664 & 27.39 & 26.88 & -0.07 & 0.20 & 0336-35 & G & 0.005 & 22.82 & 20.41 & -2.13 & -1.86 \\
\hline 2131-021 & BL & 0.557 & 26.87 & 26.82 & 1.19 & 1.47 & $0404+768$ & G & 0.599 & 27.42 & 25.82 & -1.31 & -1.04 \\
\hline $2200+420$ & $\mathrm{BL}$ & 0.069 & 25.77 & 25.07 & -0.33 & -0.05 & $0405-123$ & G & 0.574 & 27.39 & 26.60 & -0.44 & -0.16 \\
\hline $2201+044$ & BL & 0.028 & 24.10 & 23.41 & -0.31 & -0.04 & $0406-244$ & G & 2.440 & 27.64 & 25.41 & -1.95 & -1.67 \\
\hline $2240-260$ & BL & 0.774 & 26.87 & 26.73 & 0.70 & 0.97 & $0411+141$ & G & 0.206 & 26.51 & 24.17 & -2.06 & -1.79 \\
\hline $0007+106$ & G & 0.090 & 25.33 & 23.85 & -1.19 & -0.91 & 0417-181 & G & 2.773 & 27.63 & 24.72 & -2.63 & -2.36 \\
\hline $0007+124$ & G & 0.110 & 25.41 & 22.74 & -2.39 & -2.12 & $0439+083$ & G & 0.152 & 25.07 & 23.12 & -1.67 & -1.39 \\
\hline 0015-229 & G & 2.010 & 27.42 & 25.32 & -1.82 & -1.54 & $0445+44$ & G & 0.021 & 24.58 & 21.92 & -2.38 & -2.11 \\
\hline $0019+230$ & G & 0.134 & 24.63 & 22.67 & -1.68 & -1.40 & $0448+520$ & G & 0.109 & 26.79 & 23.57 & -2.94 & -2.67 \\
\hline $0031+060$ & G & 0.133 & 24.71 & 22.48 & -1.95 & -1.67 & $0449-175$ & G & 0.031 & 23.97 & 22.03 & -1.66 & -1.38 \\
\hline $0034+254$ & G & 0.032 & 23.13 & 21.77 & -1.06 & -0.79 & $0457+052$ & G & 0.098 & 24.48 & 22.97 & -1.22 & -0.94 \\
\hline 0034-014 & G & 0.073 & 25.41 & 23.48 & -1.65 & -1.37 & $0457+054$ & G & 0.054 & 23.71 & 21.81 & -1.62 & -1.34 \\
\hline $0035+180$ & G & 0.145 & 24.70 & 23.27 & -1.14 & -0.86 & $0459+252$ & G & 0.278 & 26.72 & 25.33 & -1.10 & -0.82 \\
\hline $0039+211$ & G & 0.102 & 24.89 & 23.60 & -0.99 & -0.71 & $0518-458$ & G & 0.034 & 25.93 & 24.01 & -1.64 & -1.36 \\
\hline $0040+517$ & G & 0.174 & 26.52 & 23.07 & -3.17 & -2.90 & 0546-329 & G & 0.147 & 25.47 & 23.90 & -1.28 & -1.01 \\
\hline $0043+201$ & G & 0.106 & 25.06 & 23.07 & -1.71 & -1.43 & $0658+329$ & G & 0.127 & 24.78 & 22.87 & -1.63 & -1.35 \\
\hline $0047+241$ & G & 0.082 & 24.17 & 23.08 & -0.78 & -0.50 & $0658+330$ & G & 0.127 & 24.98 & 23.68 & -1.00 & -0.72 \\
\hline $0050-220$ & G & 0.059 & 23.57 & 22.98 & -0.18 & 0.09 & $0702+749$ & G & 0.292 & 26.41 & 24.00 & -2.13 & -1.86 \\
\hline $0053+260$ & G & 0.192 & 25.04 & 23.00 & -1.76 & -1.48 & $0704+351$ & G & 0.078 & 24.28 & 21.83 & -2.17 & -1.90 \\
\hline 0053-016 & G & 0.042 & 24.34 & 22.19 & -1.87 & -1.59 & $0712+534$ & G & 0.064 & 24.83 & 22.96 & -1.59 & -1.31 \\
\hline $0055+265$ & G & 0.047 & 24.61 & 22.29 & -2.04 & -1.77 & $0720+381$ & G & 0.220 & 25.24 & 24.92 & 0.24 & 0.52 \\
\hline 0055-016 & G & 0.045 & 25.10 & 23.32 & -1.50 & -1.22 & $0720+670$ & G & 0.086 & 23.85 & 23.58 & 0.34 & 0.62 \\
\hline $0104+321$ & G & 0.017 & 24.21 & 22.45 & -1.48 & -1.20 & $0733+597$ & G & 0.040 & 23.99 & 22.93 & -0.74 & -0.47 \\
\hline 0108-146 & G & 0.103 & 24.60 & 23.80 & -0.45 & -0.17 & $0738+441$ & G & 0.117 & 24.92 & 23.86 & -0.74 & -0.47 \\
\hline $0110+152$ & G & 0.048 & 24.34 & 22.06 & -2.00 & -1.72 & $0745+521$ & G & 0.068 & 24.42 & 22.68 & -1.46 & -1.18 \\
\hline 0114-476 & G & 0.146 & 25.87 & 23.16 & -2.43 & -2.16 & $0755+379$ & G & 0.041 & 24.49 & 23.59 & -0.57 & -0.29 \\
\hline 0123-016 & G & 0.019 & 24.38 & 22.02 & -2.08 & -1.81 & $0756+272$ & G & 0.096 & 24.66 & 22.68 & -1.70 & -1.42 \\
\hline $0124+189$ & G & 0.042 & 24.42 & 22.59 & -1.55 & -1.27 & $0757+395$ & G & 0.057 & 23.60 & 23.16 & 0.03 & 0.31 \\
\hline $0128+002$ & G & 0.103 & 24.82 & 23.13 & -1.40 & -1.13 & $0821+695$ & G & 0.538 & 25.70 & 24.36 & -1.04 & -0.77 \\
\hline $0136+185$ & G & 0.069 & 23.92 & 22.44 & -1.19 & -0.91 & $0824+294$ & G & 0.458 & 26.73 & 24.96 & -1.49 & -1.21 \\
\hline $0136+396$ & G & 0.211 & 25.76 & 24.29 & -1.18 & -0.90 & $0828+193$ & G & 2.572 & 26.97 & 25.68 & -0.99 & -0.71 \\
\hline $0139+073$ & G & 0.062 & 23.26 & 22.31 & -0.62 & -0.35 & $0831+557$ & G & 0.240 & 26.97 & 25.45 & -1.23 & -0.95 \\
\hline $0141+061$ & G & 0.089 & 24.04 & 22.25 & -1.51 & -1.23 & $0836+290$ & G & 0.065 & 24.73 & 23.86 & -0.53 & -0.25 \\
\hline $0149+358$ & G & 0.016 & 22.62 & 21.31 & -1.01 & -0.74 & $0838+325$ & $\mathrm{G}$ & 0.069 & 24.51 & 24.13 & 0.13 & 0.41 \\
\hline
\end{tabular}


J. H. Fan and J. S. Zhang: The core dominance parameter of extragalactic radio sources, Online Material p. 3

Table 1. continued.

\begin{tabular}{|c|c|c|c|c|c|c|c|c|c|c|c|c|c|}
\hline $\begin{array}{l}\text { Name } \\
\text { (1) }\end{array}$ & $\begin{array}{l}\text { ID } \\
\text { (2) }\end{array}$ & $\begin{array}{c}z \\
(3)\end{array}$ & $\begin{array}{c}\log P^{\mathrm{T}} \\
\text { (4) }\end{array}$ & $\begin{array}{c}\log P^{\mathrm{C}} \\
\text { (5) }\end{array}$ & $\begin{array}{c}\log R \\
\text { (6) }\end{array}$ & $\begin{array}{c}\log R \\
\text { (7) }\end{array}$ & $\begin{array}{l}\text { Name } \\
\text { (1) }\end{array}$ & $\begin{array}{l}\text { ID } \\
\text { (2) }\end{array}$ & $\begin{array}{c}z \\
(3)\end{array}$ & $\begin{array}{c}\log P^{\mathrm{T}} \\
\text { (4) }\end{array}$ & $\begin{array}{c}\log P^{\mathrm{C}} \\
(5)\end{array}$ & $\begin{array}{c}\log R \\
\text { (6) }\end{array}$ & $\begin{array}{c}\log R \\
\text { (7) }\end{array}$ \\
\hline $0844+319$ & $\mathrm{G}$ & 0.068 & 24.88 & 23.35 & -1.24 & -0.96 & $1256+281$ & $\mathrm{G}$ & 0.024 & 22.91 & 21.08 & -1.55 & -1.27 \\
\hline $0844+540$ & G & 0.045 & 24.56 & 22.59 & -1.69 & -1.41 & $1257+28$ & G & 0.023 & 23.05 & 20.81 & -1.96 & -1.68 \\
\hline $0902+343$ & G & 3.395 & 27.94 & 26.33 & -1.32 & -1.05 & $1258-321$ & G & 0.017 & 23.53 & 22.54 & -0.67 & -0.39 \\
\hline 0905-097 & G & 0.054 & 24.15 & 23.16 & -0.67 & -0.39 & $1306+107$ & G & 0.136 & 24.33 & 23.12 & -0.91 & -0.63 \\
\hline 0907-091 & G & 0.138 & 24.24 & 23.26 & -0.66 & -0.38 & $1308-441$ & G & 0.051 & 24.55 & 23.81 & -0.38 & -0.10 \\
\hline $0908+376$ & G & 0.105 & 24.89 & 23.50 & -1.10 & -0.82 & $1313+073$ & G & 0.051 & 24.75 & 22.57 & -1.90 & -1.62 \\
\hline 0908-103 & G & 0.129 & 25.06 & 23.39 & -1.38 & -1.11 & $1316+299$ & G & 0.073 & 24.85 & 23.25 & -1.31 & -1.04 \\
\hline $0909+162$ & G & 0.085 & 24.17 & 21.99 & -1.90 & -1.62 & $1318-434$ & G & 0.011 & 23.87 & 22.88 & -0.67 & -0.39 \\
\hline $0910+411$ & G & 0.442 & 24.51 & 23.60 & -0.58 & -0.30 & $1319+428$ & G & 0.079 & 25.27 & 22.69 & -2.30 & -2.03 \\
\hline $0913+38$ & G & 0.071 & 24.27 & 22.48 & -1.51 & -1.23 & $1320+584$ & G & 0.193 & 25.15 & 23.56 & -1.30 & -1.03 \\
\hline $0915+32$ & G & 0.062 & 24.00 & 22.56 & -1.15 & -0.87 & $1321+31$ & G & 0.016 & 23.85 & 21.77 & -1.80 & -1.52 \\
\hline 0936-041 & G & 0.094 & 24.70 & 23.16 & -1.25 & -0.97 & $1322+36$ & G & 0.018 & 23.42 & 22.38 & -0.72 & -0.45 \\
\hline $1004+146$ & G & 0.031 & 24.07 & 22.97 & -0.79 & -0.51 & $1322-427$ & G & 0.001 & 24.62 & 22.12 & -2.22 & -1.95 \\
\hline $1005+28$ & G & 0.148 & 24.25 & 22.60 & -1.36 & -1.09 & $1333-337$ & G & 0.081 & 25.23 & 23.61 & -1.33 & -1.06 \\
\hline $1009+748$ & G & 0.810 & 27.27 & 24.31 & -2.68 & -2.41 & $1335+047$ & G & 0.013 & 24.41 & 22.46 & -1.67 & -1.39 \\
\hline $1010+350$ & G & 1.414 & 27.14 & 26.87 & 0.34 & 0.62 & $1336+39$ & G & 0.246 & 26.39 & 23.85 & -2.26 & -1.99 \\
\hline $1029+570$ & G & 0.034 & 23.81 & 22.50 & -1.01 & -0.74 & $1343-601$ & G & 0.012 & 25.20 & 23.58 & -1.33 & -1.06 \\
\hline $1040+31$ & G & 0.036 & 24.03 & 22.77 & -0.96 & -0.68 & $1350+316$ & G & 0.045 & 25.01 & 22.70 & -2.03 & -1.76 \\
\hline $1100+358$ & G & 1.440 & 27.06 & 25.35 & -1.43 & -1.15 & $1355+219$ & G & 0.067 & 23.74 & 23.47 & 0.34 & 0.62 \\
\hline $1101-325$ & G & 0.355 & 26.28 & 25.82 & 0.00 & 0.28 & $1357+28$ & G & 0.063 & 24.03 & 22.45 & -1.29 & -1.02 \\
\hline $1102+30$ & G & 0.072 & 24.29 & 22.76 & -1.24 & -0.96 & $1359-113$ & G & 0.037 & 24.49 & 23.08 & -1.12 & -0.84 \\
\hline $1107-372$ & G & 0.010 & 22.80 & 21.42 & -1.09 & -0.81 & $1400-001$ & G & 2.363 & 27.29 & 25.51 & -1.50 & -1.22 \\
\hline $1108+27$ & G & 0.033 & 23.01 & 22.21 & -0.45 & -0.17 & $1407+177$ & G & 0.016 & 23.68 & 22.00 & -1.39 & -1.12 \\
\hline $1108+411$ & G & 0.074 & 24.73 & 22.91 & -1.54 & -1.26 & $1414+110$ & G & 0.024 & 24.43 & 22.69 & -1.46 & -1.18 \\
\hline $1113+295$ & G & 0.049 & 24.67 & 22.94 & -1.45 & -1.17 & $1415+084$ & G & 0.057 & 24.08 & 22.28 & -1.52 & -1.24 \\
\hline $1113-178$ & G & 2.239 & 27.39 & 25.66 & -1.45 & -1.17 & $1420+198$ & G & 0.272 & 26.44 & 23.74 & -2.42 & -2.15 \\
\hline $1116+28$ & G & 0.067 & 24.33 & 23.17 & -0.85 & -0.58 & $1422+26$ & G & 0.037 & 24.00 & 22.25 & -1.47 & -1.19 \\
\hline $1120+013$ & G & 0.072 & 24.12 & 22.65 & -1.18 & -0.90 & $1441+522$ & G & 0.140 & 25.05 & 23.44 & -1.32 & -1.05 \\
\hline $1122+390$ & G & 0.007 & 21.76 & 20.46 & -1.00 & -0.72 & $1448+634$ & G & 0.041 & 24.73 & 22.57 & -1.88 & -1.60 \\
\hline $1130-037$ & G & 0.063 & 24.54 & 23.85 & -0.31 & -0.04 & $1450+28$ & G & 0.127 & 24.56 & 22.56 & -1.72 & -1.44 \\
\hline $1131+493$ & G & 0.032 & 24.13 & 22.74 & -1.10 & -0.82 & $1452-517$ & G & 0.016 & 23.75 & 22.68 & -0.75 & -0.48 \\
\hline $1132+492$ & G & 0.032 & 23.73 & 22.59 & -0.83 & -0.55 & $1453+120$ & G & 0.032 & 23.65 & 22.77 & -0.54 & -0.27 \\
\hline $1137+18$ & G & 0.011 & 23.06 & 20.59 & -2.19 & -1.92 & $1508+059$ & G & 0.077 & 25.13 & 22.52 & -2.33 & -2.06 \\
\hline $1138-262$ & G & 2.156 & 27.73 & 25.49 & -1.96 & -1.68 & $1508+065$ & G & 0.082 & 24.61 & 22.98 & -1.34 & -1.07 \\
\hline $1141+354$ & G & 1.781 & 27.15 & 24.73 & -2.14 & -1.87 & $1518+045$ & G & 0.052 & 24.91 & 23.85 & -0.74 & -0.47 \\
\hline $1146+596$ & G & 0.011 & 23.71 & 21.93 & -1.50 & -1.22 & $1521+288$ & G & 0.083 & 24.58 & 23.58 & -0.68 & -0.40 \\
\hline $1155+266$ & G & 0.112 & 25.10 & 22.87 & -1.95 & -1.67 & $1525+290$ & G & 0.065 & 23.98 & 22.10 & -1.60 & -1.32 \\
\hline $1159+583$ & G & 0.102 & 25.07 & 22.81 & -1.98 & -1.70 & $1528+29$ & G & 0.084 & 24.21 & 22.31 & -1.62 & -1.34 \\
\hline $1201+282$ & G & 0.139 & 24.68 & 21.89 & -2.51 & -2.24 & $1553+24$ & G & 0.043 & 23.36 & 23.01 & 0.18 & 0.46 \\
\hline $1209+746$ & G & 0.107 & 24.99 & 23.26 & -1.45 & -1.17 & $1603+001$ & G & 0.059 & 24.91 & 23.27 & -1.35 & -1.08 \\
\hline $1216+061$ & G & 0.007 & 24.01 & 22.25 & -1.48 & -1.20 & $1603+178$ & G & 0.032 & 23.93 & 21.97 & -1.68 & -1.40 \\
\hline $1222+13$ & G & 0.003 & 23.24 & 21.72 & -1.23 & -0.95 & $1607+268$ & G & 0.473 & 26.99 & 25.78 & -0.91 & -0.63 \\
\hline $1227+119$ & G & 0.084 & 25.12 & 23.34 & -1.50 & -1.22 & $1610-608$ & G & 0.014 & 23.97 & 22.41 & -1.27 & -1.00 \\
\hline $1231+674$ & G & 0.106 & 25.06 & 22.85 & -1.93 & -1.65 & $1613+27$ & G & 0.065 & 24.03 & 22.69 & -1.04 & -0.77 \\
\hline $1233+168$ & G & 0.078 & 24.96 & 22.68 & -2.00 & -1.72 & $1615+351$ & G & 0.030 & 23.40 & 22.48 & -0.59 & -0.31 \\
\hline $1233+169$ & G & 0.078 & 24.64 & 23.60 & -0.72 & -0.45 & $1615+425$ & G & 0.131 & 24.20 & 23.20 & -0.68 & -0.40 \\
\hline $1234-723$ & G & 0.023 & 23.87 & 23.16 & -0.34 & -0.06 & $1621+380$ & G & 0.031 & 23.62 & 22.75 & -0.53 & -0.25 \\
\hline $1238+188$ & G & 0.072 & 24.48 & 22.88 & -1.31 & -1.04 & $1623+410$ & G & 0.030 & 23.11 & 22.76 & 0.18 & 0.46 \\
\hline $1243+267$ & G & 0.089 & 24.22 & 22.81 & -1.12 & -0.84 & $1626+278$ & G & 0.448 & 26.80 & 23.49 & -3.03 & -2.76 \\
\hline $1244+699$ & G & 0.157 & 24.80 & 23.25 & -1.26 & -0.98 & $1626+396$ & G & 0.030 & 24.51 & 23.16 & -1.05 & -0.78 \\
\hline $1247-012$ & G & 0.083 & 24.34 & 23.45 & -0.55 & -0.28 & $1636+379$ & G & 0.161 & 25.26 & 23.12 & -1.86 & -1.58 \\
\hline $1250-102$ & G & 0.014 & 23.27 & 22.14 & -0.82 & -0.54 & $1637+299$ & G & 0.088 & 24.40 & 22.89 & -1.22 & -0.94 \\
\hline $1251+273$ & G & 0.027 & 23.55 & 21.16 & -2.11 & -1.84 & $1638+32$ & G & 0.140 & 24.80 & 24.06 & -0.38 & -0.10 \\
\hline $1252-122$ & G & 0.086 & 25.38 & 22.99 & -2.11 & -1.84 & $1641+399$ & G & 0.110 & 24.93 & 23.21 & -1.44 & -1.16 \\
\hline $1253-055$ & G & 0.014 & 24.23 & 22.13 & -1.82 & -1.54 & $1643+27$ & G & 0.102 & 24.05 & 22.74 & -1.01 & -0.74 \\
\hline $1254+277$ & G & 0.025 & 22.63 & 21.18 & -1.16 & -0.88 & $1648+050$ & G & 0.154 & 27.10 & 23.61 & -3.21 & -2.94 \\
\hline
\end{tabular}


J. H. Fan and J. S. Zhang: The core dominance parameter of extragalactic radio sources, Online Material p. 4

Table 1. continued.

\begin{tabular}{|c|c|c|c|c|c|c|c|c|c|c|c|c|c|}
\hline $\begin{array}{l}\text { Name } \\
\text { (1) }\end{array}$ & $\begin{array}{l}\text { ID } \\
\text { (2) }\end{array}$ & $\begin{array}{c}z \\
(3)\end{array}$ & $\begin{array}{c}\log P^{\mathrm{T}} \\
\text { (4) }\end{array}$ & $\begin{array}{c}\log P^{\mathrm{C}} \\
(5)\end{array}$ & $\begin{array}{c}\log R \\
(6)\end{array}$ & $\begin{array}{c}\log R \\
\text { (7) }\end{array}$ & $\begin{array}{l}\text { Name } \\
\text { (1) }\end{array}$ & $\begin{array}{l}\text { ID } \\
(2)\end{array}$ & $\begin{array}{c}z \\
(3) \\
\end{array}$ & $\begin{array}{c}\log P^{T} \\
\text { (4) }\end{array}$ & $\begin{array}{c}\log P^{\mathrm{C}} \\
(5)\end{array}$ & $\begin{array}{c}\log R \\
(6)\end{array}$ & $\begin{array}{c}\log R \\
\text { (7) }\end{array}$ \\
\hline $1658+30 \mathrm{~A}$ & G & 0.035 & 23.88 & 22.89 & -0.67 & -0.39 & $0710+439$ & $\mathrm{~S}$ & 0.518 & 27.15 & 24.82 & -2.05 & -1.78 \\
\hline $1658+326$ & G & 0.102 & 24.52 & 22.62 & -1.62 & -1.34 & $1005+449$ & $\mathrm{~S}$ & 0.880 & 27.92 & 24.65 & -2.99 & -2.72 \\
\hline $1709+460$ & G & 0.056 & 23.32 & 21.84 & -1.19 & -0.91 & $1142+198$ & S & 0.021 & 24.48 & 23.09 & -1.10 & -0.82 \\
\hline $1712+638$ & G & 0.806 & 27.23 & 24.39 & -2.56 & -2.29 & $1358+624$ & $S$ & 0.431 & 27.01 & 24.22 & -2.51 & -2.24 \\
\hline $1736+32$ & G & 0.074 & 24.14 & 22.92 & -0.92 & -0.64 & $1416+423$ & S0.5 & 0.017 & 21.86 & 21.07 & -0.44 & -0.16 \\
\hline $1743+666$ & G & 0.272 & 25.58 & 23.55 & -1.75 & -1.47 & $0055+300$ & $\mathrm{~S} 1$ & 0.017 & 24.08 & 23.24 & -0.50 & -0.22 \\
\hline $1747+30$ & G & 0.130 & 23.96 & 22.91 & -0.73 & -0.46 & $0106+729$ & S1 & 0.181 & 26.11 & 23.76 & -2.07 & -1.80 \\
\hline $1752+32 B$ & G & 0.045 & 23.47 & 22.30 & -0.86 & -0.59 & 0240-002 & S1 & 0.004 & 22.94 & 20.99 & -1.67 & -1.39 \\
\hline $1753+580$ & G & 0.160 & 24.62 & 23.32 & -1.00 & -0.72 & $0309+390$ & S1 & 0.161 & 25.72 & 24.31 & -1.12 & -0.84 \\
\hline $1759+211$ & G & 0.080 & 24.78 & 23.11 & -1.38 & -1.11 & $0410+110$ & S1 & 0.307 & 26.80 & 25.15 & -1.36 & -1.09 \\
\hline $1815+680$ & G & 0.230 & 25.09 & 24.71 & 0.13 & 0.41 & $0415+379$ & S1 & 0.049 & 26.29 & 24.47 & -1.54 & -1.26 \\
\hline $1819+396$ & G & 0.400 & 26.85 & 25.40 & -1.16 & -0.88 & $0511+008$ & S1 & 0.127 & 25.69 & 22.41 & -3.00 & -2.73 \\
\hline $1823+568$ & G & 0.088 & 24.84 & 23.65 & -0.88 & -0.61 & 0549-074 & S1 & 0.007 & 22.18 & 21.63 & -0.13 & 0.15 \\
\hline $1827+32$ & G & 0.066 & 24.07 & 23.08 & -0.67 & -0.39 & $0609+710$ & S1 & 0.014 & 23.54 & 21.85 & -1.40 & -1.13 \\
\hline $1829+290$ & G & 0.842 & 27.47 & 25.26 & -1.93 & -1.65 & $0645+744$ & S1 & 0.019 & 23.03 & 20.81 & -1.94 & -1.66 \\
\hline $1834+620$ & G & 0.519 & 26.45 & 23.78 & -2.39 & -2.12 & $0917+458$ & S1 & 0.174 & 26.45 & 24.18 & -1.99 & -1.71 \\
\hline $1842+455$ & G & 0.091 & 25.73 & 23.76 & -1.69 & -1.41 & $0958+290$ & S1 & 0.185 & 26.30 & 24.14 & -1.88 & -1.60 \\
\hline $1850+702$ & G & 0.079 & 24.32 & 23.96 & 0.17 & 0.44 & $1208+396$ & S1 & 0.003 & 21.71 & 20.47 & -0.94 & -0.66 \\
\hline $1919+479$ & G & 0.103 & 25.22 & 23.19 & -1.75 & -1.47 & $1223+129$ & S1 & 0.008 & 22.11 & 20.16 & -1.67 & -1.39 \\
\hline $1939+605$ & G & 0.201 & 26.37 & 24.09 & -2.00 & -1.72 & $1447+771$ & S1 & 0.141 & 25.75 & 24.53 & -0.92 & -0.64 \\
\hline $1940+50$ & G & 0.025 & 24.31 & 22.08 & -1.95 & -1.67 & $1833+326$ & S1 & 0.058 & 25.34 & 23.63 & -1.43 & -1.15 \\
\hline $1946+708$ & G & 0.101 & 25.03 & 23.26 & -1.49 & -1.21 & $1845+797$ & S1 & 0.057 & 25.59 & 24.10 & -1.20 & -0.92 \\
\hline $2025-218$ & G & 2.630 & 27.65 & 24.88 & -2.49 & -2.22 & 2005-044 & S1 & 0.057 & 27.73 & 24.12 & -3.33 & -3.06 \\
\hline $2036-254$ & G & 2.000 & 27.42 & 24.29 & -2.85 & -2.58 & $2116+818$ & S1 & 0.086 & 24.51 & 24.11 & 0.10 & 0.37 \\
\hline $2045+068$ & G & 0.127 & 25.67 & 23.60 & -1.79 & -1.51 & $2223-052$ & S1 & 0.057 & 25.30 & 23.51 & -1.51 & -1.23 \\
\hline $2105+236$ & G & 2.479 & 27.63 & 25.94 & -1.40 & -1.13 & $0116+082$ & S2 & 0.594 & 27.18 & 25.54 & -1.35 & -1.08 \\
\hline $2116+26$ & G & 0.016 & 22.72 & 22.10 & -0.22 & 0.05 & $0256+366$ & $\mathrm{~S} 2$ & 0.012 & 22.18 & 21.26 & -0.59 & -0.31 \\
\hline $2128+048$ & G & 0.990 & 27.85 & 25.46 & -2.11 & -1.84 & $0328-033$ & S2 & 0.020 & 21.55 & 20.18 & -1.07 & -0.80 \\
\hline $2141+279$ & G & 0.215 & 26.21 & 23.99 & -1.94 & -1.66 & $0356+102$ & $\mathrm{~S} 2$ & 0.031 & 25.02 & 21.81 & -2.93 & -2.66 \\
\hline $2148-555$ & G & 0.035 & 26.14 & 22.91 & -2.95 & -2.68 & $0651+542$ & S2 & 0.238 & 26.35 & 23.13 & -2.94 & -2.67 \\
\hline 2149-158 & G & 0.062 & 24.26 & 22.80 & -1.17 & -0.89 & 0714-292 & S2 & 0.005 & 21.34 & 19.28 & -1.78 & -1.50 \\
\hline $2152+085$ & G & 0.150 & 24.77 & 23.28 & -1.20 & -0.92 & 0806-103 & S2 & 0.110 & 25.73 & 23.88 & -1.57 & -1.29 \\
\hline $2152-699$ & G & 0.028 & 25.82 & 23.86 & -1.68 & -1.40 & $0836+299$ & S2 & 0.064 & 24.51 & 22.97 & -1.25 & -0.97 \\
\hline $2153+377$ & G & 0.292 & 26.86 & 23.99 & -2.59 & -2.32 & $1030+602$ & $\mathrm{~S} 2$ & 0.051 & 22.67 & 21.35 & -1.02 & -0.75 \\
\hline $2202+128$ & G & 2.704 & 27.37 & 24.82 & -2.27 & -2.00 & $1201+205$ & S2 & 0.024 & 23.58 & 21.38 & -1.92 & -1.64 \\
\hline $2203+292$ & G & 0.708 & 26.96 & 23.16 & -3.52 & -3.25 & $1216+423$ & $\mathrm{~S} 2$ & 0.002 & 21.68 & 19.70 & -1.70 & -1.42 \\
\hline $2212+136$ & G & 0.027 & 24.41 & 22.10 & -2.03 & -1.76 & $1244+492$ & S2 & 0.206 & 25.84 & 24.30 & -1.25 & -0.97 \\
\hline $2229+391$ & G & 0.017 & 24.02 & 22.07 & -1.67 & -1.39 & $1345+125$ & $\mathrm{~S} 2$ & 0.121 & 26.10 & 24.87 & -0.93 & -0.65 \\
\hline $2229-086$ & G & 0.073 & 24.68 & 23.23 & -1.16 & -0.88 & $1529+242$ & S2 & 0.096 & 26.15 & 23.62 & -2.25 & -1.98 \\
\hline $2236+35$ & G & 0.028 & 23.44 & 21.83 & -1.32 & -1.05 & $1559+021$ & S2 & 0.104 & 26.10 & 23.39 & -2.43 & -2.16 \\
\hline $2236-176$ & G & 0.070 & 24.96 & 22.71 & -1.97 & -1.69 & $1637+826$ & S2 & 0.023 & 24.14 & 23.66 & -0.03 & 0.25 \\
\hline $2243+394$ & G & 0.081 & 25.90 & 24.23 & -1.38 & -1.11 & $1642+690$ & S2 & 0.160 & 26.05 & 24.81 & -0.94 & -0.66 \\
\hline $2243-178$ & G & 0.123 & 24.89 & 23.28 & -1.32 & -1.05 & $1949+023$ & S2 & 0.059 & 25.41 & 22.59 & -2.54 & -2.27 \\
\hline $2259+137$ & G & 0.172 & 24.91 & 23.74 & -0.86 & -0.59 & $2121+248$ & S2 & 0.102 & 26.15 & 22.76 & -3.11 & -2.84 \\
\hline $2308+072$ & G & 0.045 & 24.75 & 24.10 & -0.26 & 0.01 & 0238-084 & S3 & 0.005 & 22.96 & 22.43 & -0.10 & 0.17 \\
\hline $2309+184$ & G & 0.428 & 26.64 & 24.21 & -2.15 & -1.88 & 0915-118 & S3 & 0.053 & 26.14 & 23.95 & -1.91 & -1.63 \\
\hline $2316+184$ & G & 0.040 & 23.70 & 22.41 & -0.99 & -0.71 & $1003+351$ & S3 & 0.099 & 25.78 & 24.64 & -0.83 & -0.55 \\
\hline $2318+079$ & G & 0.011 & 23.17 & 21.31 & -1.58 & -1.30 & $0007+332$ & Q & 0.743 & 26.55 & 24.57 & -1.70 & -1.42 \\
\hline $2322+143$ & G & 0.044 & 24.22 & 22.25 & -1.69 & -1.41 & $0017+154$ & $\mathrm{Q}$ & 2.012 & 28.16 & 25.45 & -2.43 & -2.16 \\
\hline $2330+091$ & G & 0.162 & 24.82 & 22.56 & -1.98 & -1.70 & $0017+257$ & Q & 0.284 & 26.62 & 26.23 & 0.11 & 0.39 \\
\hline $2335+267$ & G & 0.029 & 24.85 & 23.37 & -1.19 & -0.91 & 0017-207 & Q & 0.545 & 26.26 & 23.67 & -2.31 & -2.04 \\
\hline $2337+268$ & G & 0.031 & 23.49 & 23.15 & 0.20 & 0.48 & 0022-297 & Q & 0.406 & 26.75 & 25.45 & -1.00 & -0.72 \\
\hline $2352+495$ & G & 0.238 & 26.26 & 25.74 & -0.09 & 0.19 & $0033+079$ & Q & 1.578 & 27.08 & 26.16 & -0.59 & -0.31 \\
\hline $2354+471$ & G & 0.046 & 24.63 & 22.49 & -1.86 & -1.58 & $0033+183$ & Q & 1.469 & 27.65 & 25.58 & -1.79 & -1.51 \\
\hline $2357+004$ & G & 0.084 & 25.32 & 23.65 & -1.38 & -1.11 & $0035+121$ & Q & 1.395 & 27.67 & 26.97 & -0.33 & -0.05 \\
\hline $2210+016$ & G? & 0.500 & 26.92 & 25.72 & -0.90 & -0.62 & 0038-019 & $\mathrm{Q}$ & 1.690 & 27.63 & 26.44 & -0.88 & -0.61 \\
\hline
\end{tabular}


J. H. Fan and J. S. Zhang: The core dominance parameter of extragalactic radio sources, Online Material p. 5

Table 1. continued.

\begin{tabular}{|c|c|c|c|c|c|c|c|c|c|c|c|c|c|}
\hline $\begin{array}{l}\text { Name } \\
\text { (1) }\end{array}$ & $\begin{array}{l}\text { ID } \\
(2) \\
\end{array}$ & $\begin{array}{c}z \\
(3) \\
\end{array}$ & $\begin{array}{c}\log P^{\mathrm{T}} \\
\text { (4) }\end{array}$ & $\begin{array}{c}\log P^{\mathrm{C}} \\
\text { (5) }\end{array}$ & $\begin{array}{c}\log R \\
(6) \\
\end{array}$ & $\begin{array}{c}\log R \\
(7) \\
\end{array}$ & $\begin{array}{l}\text { Name } \\
(1)\end{array}$ & $\begin{array}{l}\text { ID } \\
(2) \\
\end{array}$ & $\begin{array}{c}z \\
(3) \\
\end{array}$ & $\begin{array}{c}\log P^{T} \\
\text { (4) }\end{array}$ & $\begin{array}{c}\log P^{\mathrm{C}} \\
\text { (5) } \\
\end{array}$ & $\begin{array}{c}\log R \\
(6) \\
\end{array}$ & $\begin{array}{c}\log R \\
\text { (7) } \\
\end{array}$ \\
\hline $0048+509$ & $\mathrm{Q}$ & 0.937 & 27.30 & 24.98 & -2.04 & -1.77 & $0836+195$ & $\mathrm{Q}$ & 1.691 & 27.24 & 26.56 & -0.30 & -0.03 \\
\hline $0051+291$ & Q & 1.828 & 27.47 & 26.90 & -0.16 & 0.12 & $0836+710$ & Q & 2.160 & 28.51 & 27.67 & -0.50 & -0.22 \\
\hline $0109+200$ & Q & 0.746 & 26.97 & 25.98 & -0.67 & -0.39 & $838+133$ & Q & 0.684 & 27.23 & 26.45 & -0.42 & -0.15 \\
\hline $0110+297$ & Q & 0.363 & 26.13 & 25.04 & -0.78 & -0.50 & $0839+186$ & Q & 1.272 & 27.39 & 27.23 & 0.63 & 0.90 \\
\hline $0127+233$ & Q & 1.460 & 28.01 & 26.01 & -1.72 & -1.44 & $839+616$ & Q & 0.862 & 27.13 & 25.06 & -1.79 & -1.51 \\
\hline $0130+24$ & Q & 0.457 & 26.21 & 25.11 & -0.79 & -0.51 & $0850+140$ & Q & 1.110 & 27.52 & 26.27 & -0.95 & -0.67 \\
\hline $0133+207$ & Q & 0.425 & 26.89 & 25.23 & -1.37 & -1.10 & $0850+581$ & Q & 1.322 & 27.35 & 27.26 & 0.91 & 1.19 \\
\hline $0134+329$ & Q & 0.367 & 27.42 & 25.00 & -2.14 & -1.87 & $0855+143$ & Q & 1.049 & 27.61 & 25.38 & -1.95 & -1.67 \\
\hline $0138+136$ & Q & 0.620 & 27.14 & 24.49 & -2.37 & -2.10 & $0859+470$ & Q & 1.462 & 28.17 & 27.27 & -0.57 & -0.29 \\
\hline $0153+744$ & Q & 2.338 & 28.62 & 27.81 & -0.46 & -0.18 & $359+c+4$ & Q & 1.499 & 27.33 & 27.24 & 0.91 & 1.19 \\
\hline $0202+149$ & Q & 0.833 & 27.61 & 27.23 & 0.13 & 0.41 & 0 & Q & 1.330 & 27.99 & 27.36 & -0.24 & 0.04 \\
\hline $0212+735$ & Q & 2.367 & 28.59 & 28.20 & 0.11 & 0.39 & $03+$ & Q & 0.411 & 26.27 & 24.63 & -1.35 & -1.08 \\
\hline $0221+067$ & Q & 0.511 & 26.81 & 26.25 & -0.14 & 0.13 & $0906+430$ & Q & 0.668 & 27.37 & 26.86 & -0.07 & 0.20 \\
\hline $0229+341$ & Q & 1.238 & 27.65 & 24.37 & -3.00 & -2.73 & $0919+218$ & Q & 1.421 & 27.2 & 25.8 & -1.10 & -0.82 \\
\hline $0238+100$ & Q & 1.816 & 27.29 & 26.16 & -0.82 & -0.54 & & Q & 0.617 & 26. & 24.71 & -1.56 & -1.28 \\
\hline $0307+444$ & Q & 1.165 & 27.40 & 26.61 & -0.44 & -0.16 & $0938+$ & Q & 0.618 & 26. & 25.00 & -1.71 & -1.43 \\
\hline $0317-023$ & Q & 2.092 & 27.68 & 27.09 & -0.18 & 0.09 & $0941+2$ & Q & 2.910 & 28.12 & 27.39 & -0.36 & -0.09 \\
\hline $0333+321$ & Q & 1.258 & 28.36 & 27.23 & -0.82 & -0.54 & $0941+$ & Q & 0.565 & 26. & 26.17 & 0.10 & 0.37 \\
\hline 040 & Q & 2.109 & 28.53 & 27.5 & -0.70 & -0.42 & & Q & 0.712 & 26. & 53 & 0.73 & 1.01 \\
\hline $0409+22$ & Q & 1.215 & 27.21 & 27.06 & 0.66 & 0.94 & 3 & Q & 0.907 & 27. & 26.02 & -0.69 & -0.41 \\
\hline $0411+055$ & Q & 2.639 & 28.43 & 27.17 & -0.96 & -0.68 & & Q & 1.405 & 27. & & -0.88 & -0.61 \\
\hline 0420-014 & Q & 0.915 & 27.82 & 27.26 & -0.14 & 0.13 & $1001+226$ & Q & 0.974 & 26.94 & 25.73 & -0.91 & -0.63 \\
\hline $0429+415$ & Q & 1.023 & 28.11 & 26.26 & -1.57 & -1.29 & $1004+130$ & Q & 0.240 & 25.5 & 23.8 & -1 . & -1.48 \\
\hline 0437-244 & Q & 0.840 & 26.61 & 25.02 & -1.30 & -1.03 & & Q & 0.613 & 26. & 25.85 & -0.74 & -0.47 \\
\hline $0445+097$ & Q & 2.110 & 27.78 & 27.29 & -0.04 & 0.23 & 9 & Q & 1.226 & 27. & 27. & 1.60 & 1.88 \\
\hline $0454-220$ & Q & 0.530 & 26.89 & 25.74 & -0.84 & -0.57 & $1018+$ & Q & 0.364 & 25. & 24.89 & 0.02 & 0.29 \\
\hline $0504+030$ & Q & 2.453 & 28.11 & 27.5 & -0.16 & 0.12 & $1020+$ & Q & 1.254 & 27. & 26.7 & -0 . & -0.18 \\
\hline $18+165$ & Q & 0.759 & 27.79 & 26.4 & -1.10 & -0.82 & 1 & Q & 0.828 & 27. & 26.6 & -0 . & -0.08 \\
\hline $0528+134$ & Q & 2.070 & 28.62 & 27.97 & -0.26 & 0.01 & $1023+$ & Q & 1.699 & 27. & 26.47 & -0.58 & -0.30 \\
\hline $0537+531$ & Q & 1.275 & 27.22 & 27.21 & 1.91 & 2.19 & $228+$ & Q & 0.177 & 24. & & 0.32 & 0.60 \\
\hline $0538+498$ & Q & 0.545 & 27.95 & 26.78 & -0.86 & -0.59 & $32+$ & Q & 0.680 & 25.8 & 25.45 & 0.06 & 0.34 \\
\hline $0548+165$ & Q & 0.474 & 26.85 & 25.50 & -1.05 & -0.78 & $1038+5$ & Q & 0.677 & 26. & 26.39 & 1.19 & 1.47 \\
\hline $0553-$ & Q & 1.544 & 26.81 & 26.26 & -0.13 & 0.15 & & Q & 1.029 & 27.80 & 27.16 & -0.25 & 0.03 \\
\hline 0605-085 & Q & 0.870 & 27.86 & 27.37 & -0.04 & 0.23 & & Q & 1.111 & & 27 & -0 & 0.03 \\
\hline $0637-752$ & Q & 0.654 & 27.84 & 27.40 & 0.03 & 0.31 & 6 & Q & 0.375 & 26. & 25. & -0. & 0.03 \\
\hline $0707+476$ & Q & 1.310 & 27.81 & 27.3 & -0.07 & 0.20 & 1100 & Q & 0.311 & 26. & 25.00 & -1 . & -0.84 \\
\hline $0710+118$ & Q & 0.768 & 27.28 & 25.1 & -1.84 & -1.5 & & Q & 0.42 & 26. & 25. & -0.75 & -0.48 \\
\hline $0711+356$ & Q & 1.626 & 27.99 & 26.14 & -1.57 & -1.29 & $1104+1$ & Q & 0.632 & 26.61 & 26.30 & 0.26 & 0.54 \\
\hline $0723+679$ & Q & 0.846 & 27.39 & 26.62 & -0.41 & -0.14 & $1107+483$ & Q & 0.740 & 26.70 & 25.73 & -0.64 & -0.37 \\
\hline $0730+257$ & Q & 2.686 & 27.73 & 26.37 & -1.06 & -0.79 & $1136-135$ & $\mathrm{Q}$ & 0.554 & 27.15 & 26.27 & -0.54 & -0.27 \\
\hline $0740+380$ & Q & 1.060 & 27.34 & 25.40 & -1.66 & -1.38 & $1137+660$ & Q & 0.646 & 27.90 & 25.98 & -1.64 & -1.36 \\
\hline $0740+828$ & Q & 1.991 & 28.10 & 27.34 & -0.40 & -0.12 & $142+052$ & Q & 1.342 & 27.50 & 26.94 & -0.14 & 0.13 \\
\hline $0742+318$ & Q & 0.462 & 26.55 & 26.24 & 0.26 & 0.54 & 97 & Q & 0.334 & 26.43 & 25.85 & -0.17 & 0.11 \\
\hline $0745+241$ & Q & 0.410 & 26.56 & 25.88 & -0.30 & -0.03 & $150+812$ & Q & 1.250 & 27.76 & 27.26 & -0.06 & 0.22 \\
\hline $0748+126$ & Q & 0.889 & 27.26 & 27.2 & 1.19 & 1.47 & $1158+122$ & Q & 2.018 & 27.14 & 26.13 & -0.69 & -0.41 \\
\hline $0751+298$ & Q & 2.106 & 27.47 & 26.80 & -0.29 & -0.01 & $1206+439$ & Q & 1.400 & 27.66 & 26.08 & -1.29 & -1.02 \\
\hline $0752+258$ & Q & 0.446 & 26.03 & 24.81 & -0.92 & -0.64 & $1217+023$ & Q & 0.240 & 25.68 & 25.33 & 0.18 & 0.46 \\
\hline $0758+143$ & Q & 1.200 & 27.78 & 25.95 & -1.55 & -1.27 & $1222+216$ & Q & 0.435 & 26.64 & 26.19 & 0.02 & 0.29 \\
\hline $0800+608$ & Q & 0.689 & 26.82 & 25.35 & -1.18 & -0.90 & $1225+368$ & $\mathrm{Q}$ & 1.975 & 28.36 & 27.37 & -0.67 & -0.39 \\
\hline $0802+103$ & Q & 1.956 & 28.12 & 26.36 & -1.48 & -1.20 & $1226+023$ & Q & 0.158 & 27.14 & 26.92 & 0.46 & 0.73 \\
\hline $0805+046$ & Q & & & 27.62 & 0.32 & 0.60 & $1226+105$ & Q & 2.296 & 27.81 & 26.51 & -1.00 & -0.72 \\
\hline $0812+02$ & Q & 0.402 & 26.68 & 25.60 & -0.77 & -0.49 & $1235-182$ & Q & 2.192 & 27.34 & 26.33 & -0.69 & -0.41 \\
\hline $0812+367$ & Q & 1.025 & 27.23 & 27.10 & 0.73 & 1.01 & $1241+166$ & Q & 0.557 & 27.08 & 25.74 & -1.04 & -0.77 \\
\hline $0821+621$ & Q & & & & & 1.09 & $1250+568$ & Q & & & 25.70 & -0.58 & -0.30 \\
\hline $0833+654$ & Q & 1.112 & 27.39 & 25.69 & -1.41 & -1.14 & $1258+404$ & Q & & 27.84 & 26.21 & -1.34 & -1.07 \\
\hline $0835+580$ & Q & 1.536 & 27.86 & 25.73 & -1.85 & -1.57 & $1303-250$ & $\mathrm{Q}$ & 0.738 & 26.63 & 25.46 & -0.86 & -0.59 \\
\hline
\end{tabular}


J. H. Fan and J. S. Zhang: The core dominance parameter of extragalactic radio sources, Online Material p. 6

Table 1. continued.

\begin{tabular}{|c|c|c|c|c|c|c|c|c|c|c|c|c|c|}
\hline $\begin{array}{l}\text { Name } \\
\text { (1) } \\
\end{array}$ & $\begin{array}{l}\text { ID } \\
\text { (2) } \\
\end{array}$ & $\begin{array}{c}z \\
(3) \\
\end{array}$ & $\begin{array}{c}\log P^{\mathrm{T}} \\
\text { (4) } \\
\end{array}$ & $\begin{array}{c}\log P^{\mathrm{C}} \\
\text { (5) } \\
\end{array}$ & $\begin{array}{c}\log R \\
(6) \\
\end{array}$ & $\begin{array}{c}\log R \\
(7) \\
\end{array}$ & $\begin{array}{l}\text { Name } \\
(1)\end{array}$ & $\begin{array}{l}\text { ID } \\
(2) \\
\end{array}$ & $\begin{array}{c}z \\
(3) \\
\end{array}$ & $\begin{array}{c}\log P^{\mathrm{T}} \\
\text { (4) }\end{array}$ & $\begin{array}{c}\log P^{\mathrm{C}} \\
\text { (5) }\end{array}$ & $\begin{array}{c}\log R \\
(6) \\
\end{array}$ & $\begin{array}{c}\log R \\
\text { (7) } \\
\end{array}$ \\
\hline $1305+801$ & $\mathrm{Q}$ & 1.183 & 27.15 & 26.41 & -0.38 & -0.10 & $1732+160$ & Q & 1.270 & 27.52 & 25.40 & -1.84 & -1.56 \\
\hline $1308+182$ & Q & 1.689 & 27.32 & 26.00 & -1.02 & -0.75 & $1741+279$ & Q & 0.372 & 26.75 & 26.11 & -0.25 & 0.03 \\
\hline $1311-270$ & Q & 2.195 & 27.80 & 26.66 & -0.83 & -0.55 & $1745+624$ & Q & 3.886 & 28.37 & 28.11 & 0.36 & 0.64 \\
\hline $1315+346$ & Q & 1.050 & 26.98 & 26.76 & 0.46 & 0.73 & $1800+440$ & Q & 0.663 & 26.56 & 26.04 & -0.09 & 0.19 \\
\hline $1317+520$ & Q & 1.060 & 27.37 & 26.78 & -0.18 & 0.09 & $1802+110$ & Q & 1.132 & 27.37 & 23.63 & -3.46 & -3.19 \\
\hline $1318+113$ & Q & 2.171 & 28.35 & 26.88 & -1.18 & -0.90 & $1807+279$ & Q & 1.760 & 27.66 & 27.29 & 0.15 & 0.42 \\
\hline $1323+655$ & Q & 1.618 & 27.44 & 25.71 & -1.45 & -1.17 & $1816+475$ & Q & 2.225 & 27.73 & 26.24 & -1.20 & -0.92 \\
\hline $1328+307$ & Q & 0.849 & 28.18 & 27.88 & 0.28 & 0.55 & $1828+487$ & Q & 0.692 & 27.94 & 27.30 & -0.25 & 0.03 \\
\hline $1330+022$ & Q & 0.216 & 26.19 & 25.26 & -0.60 & -0.32 & $1830+285$ & Q & 0.594 & 26.83 & 26.25 & -0.17 & 0.11 \\
\hline $1335+552$ & Q & 1.096 & 27.11 & 26.96 & 0.66 & 0.94 & $1842+681$ & Q & 0.475 & 26.54 & 26.29 & 0.39 & 0.66 \\
\hline $1345+584$ & Q & 2.039 & 27.61 & 26.41 & -0.90 & -0.62 & $1849+670$ & Q & 0.657 & 26.93 & 26.52 & 0.08 & 0.36 \\
\hline $1347+539$ & Q & 0.978 & 27.16 & 26.77 & 0.11 & 0.39 & $1856+737$ & Q & 0.460 & 26.20 & 26.00 & 0.51 & 0.79 \\
\hline $1354+195$ & Q & 0.720 & 27.15 & 26.92 & 0.43 & 0.71 & $1857+566$ & Q & 1.595 & 27.57 & 25.99 & -1.29 & -1.02 \\
\hline $1354+258$ & Q & 2.032 & 27.32 & 26.44 & -0.54 & -0.27 & $1901+319$ & Q & 0.635 & 27.20 & 26.84 & 0.17 & 0.44 \\
\hline $1402+044$ & Q & 3.211 & 28.46 & 28.22 & 0.41 & 0.68 & $1924+507$ & Q & 1.098 & 27.04 & 26.76 & 0.32 & 0.60 \\
\hline $1418+546$ & Q & 1.440 & 28.27 & 27.01 & -0.96 & -0.68 & $1928+738$ & Q & 0.302 & 26.58 & 26.49 & 0.91 & 1.19 \\
\hline $1422+202$ & Q & 0.871 & 27.29 & 25.56 & -1.45 & -1.17 & $1954+513$ & Q & 1.223 & 27.55 & 27.53 & 1.60 & 1.88 \\
\hline $1451-375$ & Q & 0.314 & 26.36 & 26.24 & 0.77 & 1.05 & $2007+777$ & Q & 0.589 & 26.65 & 25.81 & -0.50 & -0.22 \\
\hline $1458+718$ & Q & 0.904 & 28.01 & 27.62 & 0.11 & 0.39 & $2015+657$ & Q & 2.845 & 28.17 & 27.83 & 0.20 & 0.48 \\
\hline $1508-055$ & Q & 1.180 & 28.32 & 26.88 & -1.15 & -0.87 & $2037+511$ & Q & 1.686 & 28.41 & 28.18 & 0.43 & 0.71 \\
\hline $1509+158$ & Q & 0.828 & 26.96 & 26.08 & -0.54 & -0.27 & $2043+749$ & Q & 0.104 & 25.30 & 24.71 & -0.18 & 0.09 \\
\hline $1510-089$ & Q & 0.361 & 26.41 & 26.40 & 1.91 & 2.19 & $2120+168$ & Q & 1.805 & 27.63 & 25.64 & -1.71 & -1.43 \\
\hline $1510-089$ & Q & 2.100 & 28.76 & 28.19 & -0.16 & 0.12 & $2134+004$ & Q & 1.932 & 28.50 & 27.37 & -0.82 & -0.54 \\
\hline $1514-241$ & Q & 1.546 & 26.64 & 25.49 & -0.84 & -0.57 & $2138+826$ & Q & 2.350 & 27.95 & 27.51 & 0.03 & 0.31 \\
\hline $1522+155$ & Q & 0.628 & 26.36 & 26.24 & 0.77 & 1.05 & $2145+067$ & Q & 0.990 & 28.19 & 27.84 & 0.18 & 0.46 \\
\hline $1532+016$ & Q & 1.440 & 27.78 & 27.07 & -0.34 & -0.06 & $2149+212$ & Q & 1.534 & 27.55 & 26.56 & -0.67 & -0.39 \\
\hline $1540+180$ & Q & 1.662 & 27.93 & 26.91 & -0.70 & -0.42 & $2201+315$ & Q & 0.298 & 26.25 & 26.18 & 1.03 & 1.31 \\
\hline $1545+210$ & Q & 0.264 & 26.28 & 24.50 & -1.50 & -1.22 & $2209+080$ & Q & 0.484 & 26.52 & 25.83 & -0.31 & -0.04 \\
\hline $1547+215$ & Q & 1.206 & 27.87 & 23.58 & -4.01 & -3.74 & $2209+152$ & Q & 1.502 & 26.85 & 25.98 & -0.53 & -0.25 \\
\hline $1555+332$ & Q & 0.942 & 26.38 & 23.98 & -2.12 & -1.85 & $2213-283$ & Q & 0.946 & 27.00 & 25.83 & -0.86 & -0.59 \\
\hline $1602-001$ & Q & 1.625 & 27.66 & 27.01 & -0.26 & 0.01 & $2221-023$ & Q & 0.879 & 26.24 & 24.90 & -1.04 & -0.77 \\
\hline $1606+180$ & Q & 0.346 & 26.28 & 24.34 & -1.66 & -1.38 & $2230+114$ & Q & 1.037 & 28.04 & 27.68 & 0.17 & 0.44 \\
\hline $1611+343$ & Q & 1.401 & 27.88 & 27.78 & 0.86 & 1.14 & $2248+192$ & Q & 1.806 & 27.55 & 25.82 & -1.45 & -1.17 \\
\hline $1618+177$ & Q & 0.555 & 26.97 & 25.78 & -0.88 & -0.61 & $2249+185$ & Q & 1.760 & 28.04 & 27.02 & -0.70 & -0.42 \\
\hline $1622+238$ & Q & 0.927 & 27.31 & 25.56 & -1.47 & -1.19 & $2251+134$ & Q & 0.673 & 26.88 & 26.51 & 0.15 & 0.42 \\
\hline $1622-297$ & Q & 0.815 & 27.26 & 27.18 & 0.97 & 1.25 & $2251+158$ & Q & 0.859 & 28.10 & 28.03 & 1.03 & 1.31 \\
\hline $1624+416$ & Q & 2.550 & 28.57 & 27.99 & -0.17 & 0.11 & $2252+129$ & Q & 0.555 & 26.79 & 23.76 & -2.75 & -2.48 \\
\hline $1629+680$ & Q & 2.475 & 28.17 & 27.69 & -0.03 & 0.25 & 2300-189 & Q & 0.129 & 25.44 & 24.90 & -0.12 & 0.16 \\
\hline $1633+382$ & Q & 1.814 & 28.19 & 28.01 & 0.57 & 0.84 & $2305+18$ & Q & 0.313 & 26.08 & 24.78 & -1.00 & -0.72 \\
\hline $1638+398$ & Q & 1.666 & 27.66 & 27.54 & 0.77 & 1.05 & $2314-116$ & Q & 0.549 & 25.99 & 25.56 & 0.05 & 0.32 \\
\hline $1656+571$ & Q & 1.290 & 27.32 & 26.99 & 0.22 & 0.50 & $2325+29$ & Q & 1.015 & 27.34 & 26.37 & -0.64 & -0.37 \\
\hline $1658+575$ & Q & 2.173 & 27.79 & 26.48 & -1.01 & -0.74 & $2335-027$ & Q & 1.072 & 27.39 & 26.63 & -0.40 & -0.12 \\
\hline $1702+298$ & Q & 1.927 & 27.93 & 26.41 & -1.23 & -0.95 & $2338+042$ & Q & 2.594 & 27.98 & 27.15 & -0.48 & -0.21 \\
\hline $1704+608$ & Q & 0.371 & 26.65 & 24.14 & -2.23 & -1.96 & $2338-290$ & Q & 0.446 & 26.04 & 25.07 & -0.64 & -0.37 \\
\hline $1705+456$ & Q & 0.648 & 26.84 & 26.25 & -0.18 & 0.09 & $2349+327$ & Q & 0.671 & 27.53 & 25.15 & -2.10 & -1.83 \\
\hline $1719+357$ & Q & 0.263 & 25.60 & 25.25 & 0.18 & 0.46 & $2353+283$ & Q & 0.731 & 26.74 & 25.58 & -0.85 & -0.58 \\
\hline $1721+343$ & Q & 0.206 & 25.84 & 25.22 & -0.22 & 0.05 & $2354+144$ & Q & 1.810 & 27.72 & 26.57 & -0.84 & -0.57 \\
\hline
\end{tabular}

